Journal of Applied ANALysis

Vol. 7, No. 1 (2001), pp. 1-22

\title{
SEMISTABLE SELFDECOMPOSABLE LAWS ON GROUPS
}

\author{
W. HAZOD and R. SHAH \\ Received October 23, 1998 and, in revised form, April 24, 2000
}

\begin{abstract}
The most prominent examples of (operator-) selfdecomposable laws on vector spaces are (operator-) stable laws. In the past (operator-) semistability - a natural generalisation — had been intensively investigated, hence the description of the intersection of the classes of semistable and selfdecomposable laws turned out to be a challenging problem, which was finally solved by A. Łuczak's investigations [17].

For probabilities on groups, in particular on simply connected nilpotent Lie groups there exists meanwhile a satisfying theory of decomposability and semistability. Consequently it is possible to obtain a description of the intersection of these classes of measures - under additional commutativity assumptions - leading finally to partial extensions of the above-mentioned results for vector spaces to the group case.
\end{abstract}

1991 Mathematics Subject Classification. 60B15, 60E07, 60F05, 43A05.

Key words and phrases. Nilpotent Lie groups, self decomposability, semi-stability, generating functional, continuous convolution semigroup, contractive automorphisms.

The second author gratefully acknowledges the support of Alexander von Humboldt foundation while this work was done.

ISSN 1425-6908 C Heldermann Verlag. 


\section{Introduction}

The investigations of the limit behaviour of normalized products of independent group-valued random variables were quite successful during the last decades, as long as the i.i.d. case was considered. There the possible limits are - on nicely-behaved groups - embeddable into continuous convolution semigroups and it turns out that e.g. for simply connected nilpotent Lie groups the limits correspond to limit laws of operator-normalized sums on (finite dimensional) vector spaces. In particular stable and semistable laws and their domains of attraction are well understood now. (See e.g. [6], [7], [18], [20] and the references mentioned there, furthermore e.g. [10] for $\mathbb{R}^{d}$ ).

In the non-i.i.d. situation new problems arise due to the non-commutativity of the underlying group structure, the limit behaviour has to be handled within the theory of convolution hemigroups. Therefore the analogues of the important class of (operator-) selfdecomposable laws are only treated in a few publications on non-Abelian groups (see e.g. [4], [13], [14], [15], [21]). It turned out that, in contrast to the general situation in this particular case quite satisfactory results are available. Again for simply connected nilpotent Lie groups the limit behaviour is similar to the vector space situation.

Our aim is to continue and to improve these investigations: It is wellknown that for $\mathbb{R}$ or $\mathbb{R}^{d}$ semistable laws are in general not selfdecomposable, hence the characterization of the intersection of these classes of limit laws was for a long time an outstanding open problem. It is solved for vector spaces by A. Euczak in [17]. Following these investigations our aim is to show that semistable selfdecomposable laws on a simply connected nilpotent Lie group correspond to (strictly) operator-semistable selfdecomposable (or operator Lévy's) measures on the tangent space and vice versa. A first result in this direction is proved in [4] assuming an additional restrictive commutativity assumption, indeed for the more general class of semistable and strongly $\rho$-decomposable laws. We show that in special situations including the semistable selfdecomposable case - the norming operators may be chosen in such a way that this commutativity assumption is fulfilled. Thus we obtain the complete analogue of A. Łuczak's characterization for simply connected nilpotent Lie groups.

\section{Preliminaries}

Let $\mathbb{G}$ be a locally compact group, let $\mathcal{M}^{1}(\mathbb{G})$ denote the set of probabilities on $\mathbb{G}$, endowed with the topology of weak convergence. The convolution structure is defined as usual by $\int_{\mathbb{G}} f d \mu * \nu:=\int_{\mathbb{G}} \int_{\mathbb{G}} f(x y) d \mu(x) d \nu(y)$, $\mu, \nu \in \mathcal{M}^{1}(\mathbb{G}), f \in C_{0}(\mathbb{G})$. For standard notations and more informations on probabilities on groups the reader is referred e.g. to [8]. 
Definition 1.1. Let $\mu \in \mathcal{M}^{1}(\mathbb{G})$. We define the left decomposability semigroup of $\mu$ as $\mathrm{D}^{l}(\mu):=\{a \in \operatorname{End}(\mathbb{G}): \mu=\nu * a(\mu)$ for some $\nu \in$ $\left.\mathcal{M}^{1}(\mathbb{G})\right\}$. For fixed $\mu$ and $a \in \mathrm{D}^{l}(\mu)$ the set of left cofactors is defined by $\operatorname{Cof}_{\mu}^{l}(a)=\operatorname{Cof}^{l}(a):=\left\{\nu \in \mathcal{M}^{1}(\mathbb{G}): \mu=\nu * a(\mu)\right\}$.

Analogously we define the right decomposability semigroup $\mathrm{D}^{r}(\mu)$ and $\mathrm{D}^{c}(\mu):=\mathrm{D}^{l}(\mu) \cap \mathrm{D}^{r}(\mu)=\left\{a \in \operatorname{End}(\mathbb{G}): \mu=\nu_{l} * a(\mu)=a(\mu) * \nu_{r}\right.$ for some $\left.\nu_{l}, \nu_{r} \in \mathcal{M}^{1}(\mathbb{G})\right\}$, as well as $\operatorname{Cof}^{r}(a)$. To simplify notations we shall frequently use the abbreviation $\mathrm{D}(\mu):=\mathrm{D}^{l}(\mu)$ and $\operatorname{Cof}(a)=\operatorname{Cof}_{\mu}(a):=$ $\operatorname{Cof}_{\mu}^{l}(a)$.

In this paper we are mostly concerned with commuting cofactors, i.e. we consider the subsets $\mathrm{C}(\mu):=\{a: \mu=\nu * a(\mu)=a(\mu) * \nu\} \subseteq \mathrm{D}^{c}(\mu)$ and $\operatorname{Cof}_{\mu}^{c}(a):=\operatorname{Cof}_{\mu}^{l}(a) \cap \operatorname{Cof}_{\mu}^{r}(a)$. Furthermore, let $\mathrm{DC}(\mu):=\bigcap_{n \in \mathbb{N}} \mathrm{D}^{c}\left(\mu^{n}\right)$. Note that obviously we have $\mathrm{C}(\mu) \subseteq \mathrm{DC}(\mu) \subseteq \mathrm{D}^{c}(\mu)$.

On Abelian groups, in particular on vector spaces this distinction is superfluous, but our results for non-Abelian groups will depend heavily on this commutativity assumption.

Note that on vector spaces $\mathbb{G}=\mathbb{V}$, if $\mu$ is infinitely divisible, the zero set of the Fourier transform is empty and hence the cofactors are uniquely determined by $\widehat{\nu}=\widehat{\mu} \cdot(a(\widehat{\mu}))^{-1}$. This is also the case on general groups if the (operator-valued) Fourier transforms or convolution operators are injective. See e.g. [13] and [14] where injectivity is supposed, see also [15] for simply connected nilpotent Lie groups.

By abuse of language we sometimes adapt the notation $\nu(a)$ for cofactors, regardless if uniquely determined or not.

Definition 1.2. Let $\rho_{\bullet}:=\left(\rho_{t}\right)_{t \in \mathbb{R}} \subseteq$ Aut $(\mathbb{G})$ be a continuous automorphism group with additive parametrisation, $\rho_{t} \rho_{s}=\rho_{t+s}$. Let $\rho \in \operatorname{Aut}(\mathbb{G})$. A probability $\mu \in \mathcal{M}^{1}(\mathbb{G})$ is called $\rho$-decomposable if $\rho \in \mathrm{D}(\mu)$, and $\mu$ is called $\rho_{\bullet}$-decomposable if $\left\{\rho_{t}\right\}_{t \geq 0} \subseteq \mathrm{D}(\mu)$, i.e. if $\mu$ is $\rho_{t}$-decomposable for all $t \geq 0$.

It is important to assume in addition that $\rho$ (resp. $\left.\rho_{\bullet}\right)$ contracts $\mu$, i.e. $\rho^{n}(\mu) \stackrel{n \rightarrow \infty}{\longrightarrow} \varepsilon_{e}$ (resp. $\left.\rho_{t}(\mu) \stackrel{t \rightarrow \infty}{\longrightarrow} \varepsilon_{e}\right)$. In this case we call $\mu$ strongly $\rho$ decomposable (resp. strongly $\rho_{\bullet}$-decomposable or selfdecomposable). I.e. we call $\mu \in \mathcal{M}^{1}(\mathbb{G}) \rho_{\bullet}$-selfdecomposable if there exists a continuous additive one-parameter group

$$
\rho_{\bullet}=\left(\rho_{t}\right)_{t \in \mathbb{R}} \subseteq \operatorname{Aut}(\mathbb{G}) \text { such that }\left(\rho_{t}\right)_{t \geq 0} \subseteq \mathrm{D}(\mu) \text { and } \rho_{t}(\mu) \stackrel{t \rightarrow \infty}{\longrightarrow} \varepsilon_{e}
$$

This is in particular the case if $\rho^{k}(x) \stackrel{k \rightarrow \infty}{\longrightarrow} e\left(\right.$ resp. $\left.\rho_{t}(x) \stackrel{t \rightarrow \infty}{\longrightarrow} e\right), x \in \mathbb{G}$ - a condition which will be supposed mostly in the sequel: 
Throughout we use the following notation: $\rho$ (resp. $\rho_{\bullet}$ ) is contracting on $\mathbb{G}$ or contractive if $\rho^{k}(x) \stackrel{k \rightarrow \infty}{\longrightarrow} e$ (resp. $\left.\rho_{t}(x) \stackrel{t \rightarrow \infty}{\longrightarrow} e\right), x \in \mathbb{G}$.

On vector spaces (finite or infinite dimensional) strongly $\rho$-decomposable laws are called strongly operator-decomposable, see [11], [23], [24]. For the real line such laws were first studied in [16]. Strongly $\rho_{\bullet}$-decomposable laws on $\mathbb{R}^{d}$ are called operator-selfdecomposable, equivalently — under fullness assumption - measures of operator Lévy class. (See e.g. [10], [17], [25] and the literature mentioned there. For groups only a few investigations are available: [13], [14], [15], [4]; see in particular [21], [1] for related limit laws.)

Proposition 1.3. a) Let $\mu$ be strongly $\rho$-decomposable with cofactors $\nu(k) \in$ $\operatorname{Cof}\left(\rho^{k}\right)$. Then $\nu(k) \stackrel{k \rightarrow \infty}{\longrightarrow} \mu$ (for any selection of cofactors).

b) Let $\mu$ be $\rho_{\bullet}$-selfdecomposable and $\nu(t) \in \operatorname{Cof}_{\mu}\left(\rho_{t}\right)$, i.e. assume $\mu=$ $\nu(t) * \rho_{t}(\mu), t \geq 0$. Then $\nu(t) \stackrel{t \rightarrow \infty}{\longrightarrow} \mu$.

c) Conversely, let $\mathbb{G}$ be aperiodic, i.e. without non-trivial compact subgroups. Let $\left(\rho_{t}\right)$ be a continuous group $\subseteq$ Aut $(\mathbb{G})$. Furthermore, let $t \mapsto \nu(t) \in \mathcal{M}^{1}(\mathbb{G})$ be a continuous solution of the cocycle equation $\nu(t+s)=$ $\nu(s) * \rho_{s}(\nu(t))$.

If $\nu(t) \stackrel{t \rightarrow \infty}{\longrightarrow} \mu$ then $\mu$ is selfdecomposable with $\left(\rho_{t}\right)_{t \geq 0} \subseteq \mathrm{D}(\mu)$ and $\nu(t) \in$ $\operatorname{Cof}_{\mu}\left(\rho_{t}\right)$. In particular, $\rho_{t}(\mu) \rightarrow \varepsilon_{e}, t \rightarrow \infty$.

Analogous results are obtained for right cofactors and right decomposability semigroups (resp. for $\mathrm{DC}(\mu)$ ), and for commuting cofactors and $\mathrm{C}(\mu)$.

a) and b) are obvious consequences of the factor compactness theorem ([19]) respecting the contractivity of $\rho$ and $\rho_{\bullet}$.

c) Let $s>0, t>0$. Then $\nu(t+s)=\nu(s) * \rho_{s}(\nu(t))$, whence $\lim _{t \rightarrow \infty} \nu(t+s)=$ $\mu=\nu(s) * \rho_{s}(\mu)$ follows for any fixed $s . \nu(s) \stackrel{s \rightarrow \infty}{\longrightarrow} \mu$ implies, again by factor compactness, that $\mu=\mu * \mu^{\prime}$ for any accumulation point $\mu^{\prime} \in$ $\operatorname{LIM}\left(\rho_{s}(\mu)_{s \rightarrow \infty}\right)$, and therefore, since $\mathbb{G}$ is aperiodic, $\mu^{\prime}=\varepsilon_{e}$ follows. Whence the second assertion.

Throughout LIM will denote the set of accumulation points.

\section{Semistable strongly $\rho$-decomposable laws on groups}

Let $\mathbb{G}$ be a connected locally compact group. Let $\mu \in \mathcal{M}^{1}(\mathbb{G})$ be full, i.e. the support of $\mu$ is not contained in a proper closed connected subgroup. Let $\rho \in \operatorname{Aut}(\mathbb{G})$ be contracting. Let $\mu$ be strongly $\rho$-decomposable (and hence $\mathrm{D}(\mu)$ contains the discrete contracting semigroup $\left.\left\{\rho^{k}: k \in \mathbb{N}\right\}\right)$. 
For sake of completeness let us repeat the following

Definition. A continuous convolution semigroup $\left(\mu_{t}\right)_{t>0}=\mu_{\bullet}$ is called (strictly) semistable w.r.t. $a \in \operatorname{Aut}(\mathbb{G})$ and $\alpha \in(0,1)$ - in short: $(a, \alpha)$ semistable - if $a\left(\mu_{t}\right)=\mu_{\alpha t}, t \geq 0$.

It is proved in [4] that on simply connected nilpotent Lie groups $\mathbb{G}$, a semistable continuous convolution semigroup $\mu_{\bullet}$ is uniquely determined by a single measure $\mu:=\mu_{1}$. Hence semistability may be considered as a property of a single measure $\mu$ as well as of a continuous convolution semigroup $\mu_{\bullet}$.

Remark. Let $\operatorname{Inv}(\mu)$ denote the invariance group $\operatorname{Inv}(\mu):=\{a \in \operatorname{Aut}(\mathbb{G})$ : $a(\mu)=\mu\}$. It turns out to be essential that for simply connected nilpotent Lie groups semistable laws $\mu$ not only generate uniquely determined continuous convolution semigroups $\mu_{\bullet}$ with $\mu=\mu_{1}$, but moreover, the invariance groups $\operatorname{Inv}\left(\mu_{t}\right)$ do not depend on $t>0$, cf. [4].

Let $\mu_{\bullet}$ be $(a, \alpha)$-semistable. Then $\mu=\mu_{1}$ is strongly $a$-decomposable with commuting cofactors. [Indeed, let $\left(\mu_{t}\right)$ be the embedding continuous convolution semigroup with $\mu=\mu_{1}$. Then $\mu=\mu_{1}=\mu_{1-\alpha} * \mu_{\alpha}=\mu_{1-\alpha} *$ $a(\mu)=a(\mu) * \mu_{1-\alpha}$. Hence $a \in \mathrm{C}(\mu)$ and $\mu_{1-\alpha} \in \operatorname{Cof}_{\mu}^{c}(a)$. Moreover, $a$ is contracting since $\mu$ is full. $]$

On the other hand, if $\mu$ is selfdecomposable with $\left(\rho_{t}\right)_{t \geq 0} \subseteq \mathrm{D}(\mu)$ (resp. $\left.\left(\rho_{t}\right)_{t \geq 0} \subseteq \mathrm{D}^{r}(\mu)\right)$ then $\mu$ is $\rho_{t}$-decomposable for any $t \geq 0$. But even on the real line semistable laws are in general not selfdecomposable and vice versa. In the following we obtain a description of full $(a, \alpha)$-semistable and strongly $\rho$-decomposable laws in analogy to the description obtained by A. Euczak [17] for operator-semistable selfdecomposable laws on $\mathbb{R}^{d}$. On groups we have to suppose the additional restrictive commutativity assumption

$$
a \rho a^{-1} \in \rho \cdot \operatorname{Inv}(\mu) .
$$

Let $i_{a}$ denote the inner automorphism $\rho \mapsto a \rho a^{-1}$ induced by $a$ on $\operatorname{Aut}(\mathbb{G})$. Then $(*)$ is equivalent to $i_{a}(\rho) \in \rho \cdot \operatorname{Inv}(\mu)$. Since $i_{a}(\operatorname{Inv}(\mu))=$ $\operatorname{Inv}(\mu)$ this yields $i_{a^{n}}\left(\rho^{k}\right) \in \rho^{k} \cdot \operatorname{Inv}(\mu)$ for all $k, n \in \mathbb{Z}$. (Note that $i_{a}^{n}=i_{a^{n}}$.) Therefore, according to the remark above we have $a^{n} \rho^{k} \mu=\rho^{k} \mu_{\alpha^{n}}$.

We start with a result proved in [4]. To make the paper more selfcontained we include a proof.

First we need the following

Definition 2.1. Let $\mathbb{G}$ be a locally compact group, let $\mu_{\bullet}=\left(\mu_{t}\right)_{t \geq 0}$ be a continuous convolution semigroup in $\mathcal{M}^{1}(\mathbb{G})$. Then the generating functional $A$ is defined as

$$
\langle A, f\rangle:=\left.\frac{d^{+}}{d t}\left\langle\mu_{t}, f\right\rangle\right|_{t=0}=\lim _{t \searrow 0} \frac{1}{t}\left(\left\langle\mu_{t}, f\right\rangle-\left\langle\varepsilon_{e}, f\right\rangle\right)
$$


for test functions $f \in \mathcal{E}(\mathbb{G})$. (For Lie groups $\mathbb{G}$ the appropriate space of test functions is just $\mathcal{E}(\mathbb{G})=C_{b}^{\infty}(\mathbb{G})$.) For details see e.g. [3], [8].

By $\mathcal{G F}(\mathbb{G})$ we denote the set of generating functionals $\subseteq(\mathcal{E}(\mathbb{G}))^{\prime}$. For $A \in \mathcal{G F}(\mathbb{G})$ and corresponding continuous convolution semigroup $\mu_{\bullet}$ we use the notation $\operatorname{Exp}(t A):=\mu_{t}, t \geq 0$.

For non-Abelian groups generating functionals play the role of the second characteristic function (logarithm of the Fourier transform) in case of vector spaces. In fact, if $\mathbb{G}=\mathbb{R}^{d}$ then $\widehat{\mu}_{t}=e^{t \cdot \widehat{A}}, t \geq 0$, where $\widehat{A}$ denotes the Fourier transform of the linear functional $A \in\left(C_{b}^{\infty}\right)^{\prime}$. By the theorem of Hille-Yosida, also in the non-Abelian situation, generating functionals $A$ determine uniquely the convolution semigroup $\mu_{\bullet}$ :

Let $\mathcal{D}(\mathbb{G})$ denote the Schwartz-Bruhat functions - in case of Lie groups $\mathcal{D}(\mathbb{G})=C_{c}^{\infty}(\mathbb{G})$ - then $f \mapsto A * f$ extends uniquely to the infinitesimal generator of the $C_{0}$-contraction semigroup of convolution operators $f \mapsto$ $\mu_{t} * f$ on $C_{0}(\mathbb{G})$. For details the reader is referred to [8], [3].

The action of Aut $(\mathbb{G})$ on measures is canonically extended: We define $a(A)$ for $a \in \operatorname{Aut}(\mathbb{G}), A \in \mathcal{G F}(\mathbb{G})$ by

$$
\langle a(A), f\rangle:=\langle A, f \circ a\rangle \text { for } f \in \mathcal{E}(\mathbb{G}) .
$$

$\mu_{\bullet}$ is $(a, \alpha)$-semistable iff the generating functional $A$ fulfils the relation $a(A)=\alpha \cdot A$. We call $A$ semistable generating functional then.

Let $\mathbb{G}$ denote a simply connected nilpotent Lie group. Then we have

Theorem 2.2. Let $\mu$ be a full probability law on $\mathbb{G}$ which is $(a, \alpha)$-semistable and strongly $\rho$-decomposable with contracting automorphism $\rho \in \mathrm{D}^{l}(\mu)$ (resp. $\rho \in \mathrm{D}^{r}(\mu)$ ). Let $\mu_{\bullet}$ denote the corresponding semistable continuous convolution semigroup with generating functional $A$ and put $\mu_{1}=\mu$.

Assume further $\quad(*) \quad a \rho a^{-1} \in \rho \operatorname{Inv}(\mu)$.

a) Then we obtain for $k \in \mathbb{N}: \quad A=C(k)+\rho^{k}(A)$, where $C(k) \in \mathcal{G} \mathcal{F}(\mathbb{G})$ is an $(a, \alpha)$-semistable generating functional.

b) Conversely, assume $A=C(k)+\rho^{k}(A), k \in \mathbb{N}$, where $C(k) \in \mathcal{G} \mathcal{F}(\mathbb{G})$ is an $(a, \alpha)$-semistable generating functional and $\rho$ is contracting, then $A$ generates an $(a, \alpha)$-semistable continuous convolution semigroup $\mu_{t}:=\operatorname{Exp}(t A)_{t \geq 0}$.

c) If (as for vector spaces) in a) the convolution semigroups generated by $\rho^{k}(A)$ and $C(k)$ commute for $t \geq 0$, i.e. if $\rho^{k}(\operatorname{Exp}(t A))$ and $\operatorname{Exp}(t C(k))$ commute, then $\nu\left(\rho^{k}\right)=\operatorname{Exp} C(k)$ are (commuting) $(a, \alpha)$-semistable cofactors of $\rho^{k}, k \in \mathbb{N}$.

d) Conversely, let $\mu$ be strongly $\rho$-decomposable with contracting $\rho \in \mathrm{D}(\mu)$ (resp. $\left.\in \mathrm{D}^{r}(\mu)\right)$. Assume for all $k \in \mathbb{N}$ that there exist cofactors $\nu\left(\rho^{k}\right) \in$ $\operatorname{Cof}_{\mu}\left(\rho^{k}\right)$ (resp. $\left.\in \operatorname{Cof}_{\mu}^{r}\left(\rho^{k}\right)\right)$ which are $(a, \alpha)$-semistable. (Commutativity is not assumed.) Then $\mu$ is $(a, \alpha)$-semistable. 
e) Analogous results are obtained for semistable selfdecomposable laws: In this case, if we suppose

$$
a \rho_{t} a^{-1}=i_{a}\left(\rho_{t}\right) \in \rho_{t} \operatorname{Inv}(\mu), \quad t>0,
$$

then $A=C(t)+\rho_{t}(A), t \in \mathbb{R}_{+}$, with $(a, \alpha)$-semistable $C(t) \in \mathcal{G F}(\mathbb{G})$.

Proof. a) Let $\rho \in \mathrm{D}(\mu)=\mathrm{D}^{l}(\mu)$. We have $\mu=\nu\left(\rho^{k}\right) * \rho^{k}(\mu), k \in \mathbb{N}$. And on the other hand $a^{n}(\mu)=\mu_{\alpha^{n}}, n \in \mathbb{Z}$, hence $\mu_{\alpha^{n}}=a^{n}\left(\nu\left(\rho^{k}\right)\right) * a^{n} \rho^{k}(\mu)=$ (according to $(*)) a^{n}\left(\nu\left(\rho^{k}\right)\right) * \rho^{k} a^{n}(\mu)=a^{n}\left(\nu\left(\rho^{k}\right)\right) * \rho^{k}\left(\mu_{\alpha^{n}}\right)$. I.e. $a^{n}\left(\nu\left(\rho^{k}\right)\right)$ is a $\rho^{k}$-cofactor of $\mu_{\alpha^{n}}, k \in \mathbb{N}, n \in \mathbb{Z}$.

Define $A_{n}:=\alpha^{-n} \cdot\left(\mu_{\alpha^{n}}-\varepsilon_{e}\right) \in \mathcal{G F}(\mathbb{G})$. Then we have $A_{n} \stackrel{n \rightarrow \infty}{\longrightarrow} A$, and the above representation yields: $A_{n}=\alpha^{-n} \cdot\left(a^{n}\left(\nu\left(\rho^{k}\right)\right)-\varepsilon_{e}\right)+a^{n}\left(\nu\left(\rho^{k}\right)\right) * \rho^{k}\left(A_{n}\right)$. $a$ is contracting since $\mu$ is full, hence $a^{n}\left(\nu\left(\rho^{k}\right)\right) \stackrel{n \rightarrow \infty}{\longrightarrow} \varepsilon_{e}$. Furthermore, $\rho^{k}\left(A_{n}\right) \stackrel{n \rightarrow \infty}{\longrightarrow} \rho^{k}(A)$, whence we conclude $A=C(k)+\rho^{k}(A)$ for some functional $C(k) \in \mathcal{E}^{\prime}(\mathbb{G}) . A_{n}, \rho^{k}\left(A_{n}\right)$ and $\alpha^{-n} \cdot\left(a^{n}\left(\nu\left(\rho^{k}\right)\right)-\varepsilon_{e}\right)$ are obviously generating functionals (of Poisson semigroups), hence the limits $A, \rho^{k}(A)$ and $C(k)$ belong to $\mathcal{G} \mathcal{F}(\mathbb{G})$.

And $(a, \alpha)$-semistability of $A$ and $(*)$ yield: $\alpha \cdot A=a(A)=a(C(k))+$ $a \rho^{k}(A)=a(C(k))+\rho^{k} a(A)=a(C(k))+\alpha \cdot \rho^{k}(A)$. On the other hand, obviously $\alpha \cdot A=\alpha \cdot C(k)+\alpha \cdot \rho^{k}(A)$, whence $a(C(k))=\alpha \cdot C(k), k \in \mathbb{N}$, follows. I. e. $C(k)$ generates an $(a, \alpha)$-semistable continuous convolution semigroup.

b) $A=C(k)+\rho^{k}(A)$ yields $C(k) \rightarrow A$, since $\rho$ is contracting. Semistability of $A$ easily follows since the set of full $(\cdot, \alpha)$-semistable laws (resp. of semistable generating functionals) is closed in $\mathcal{M}^{1}(\mathbb{G})$ (resp. in $\mathcal{G} \mathcal{F}(\mathbb{G})$ ), as easily verified via the convergence of types theorem ([5]).

c) is obvious.

d) Let $\mu=\nu\left(\rho^{k}\right) * \rho^{k}(\mu)$ and assume the cofactors $\nu\left(\rho^{k}\right)$ to be $(a, \alpha)$ semistable. $\rho$ being contracting, we conclude $\nu\left(\rho^{k}\right) \rightarrow \mu$ (for any choice of the cofactors, cf. Proposition 1.3). Semistability of $\mu$ follows again by the closedness of the set of full $(\cdot, \alpha)$-semistable laws.

e) The proof for selfdecomposable laws and for right decomposability semigroups and cofactors is similar.

For $\mathbb{G}=\mathbb{R}^{d}$, A. Euczak [17] proved for full selfdecomposable laws the existence of contractive operator semigroups $\left(\rho_{t}=e^{-t Q}\right)_{t \geq 0}$ commuting with $a$, hence in particular fulfilling $\left(*^{\prime}\right)$ above. We shall prove an analogous result in Theorem 4.9 .

Remarks 2.3. a) The following slight generalisation is easily verified: Theorem 2.2 remains true if we replace the condition

$$
(*) \quad a \rho a^{-1}=: i_{a}(\rho) \in \rho \operatorname{Inv}(\mu)\left(\text { with } i_{a}(\kappa):=a \kappa a^{-1}, \kappa \in \operatorname{Aut}(\mathbb{G})\right) \text { by }
$$


$(* *) \quad\left\{i_{a^{n}}(\rho)=a^{n} \rho a^{-n}: n \in \mathbb{N}\right\}$ is relatively compact in Aut $(\mathbb{G})$ and $(* * *) \quad \Lambda:=\operatorname{LIM}\left(i_{a^{n}}(\rho)=a^{n} \rho a^{-n}: n \in \mathbb{N}\right) \subseteq \rho \cdot \operatorname{Inv}(\mu)$.

If only the weaker condition (**) is available then the assertions hold true with $\rho$ (resp. $\left.\rho_{t}\right)$ replaced by some $\sigma \in \operatorname{LIM}\left(i_{a^{n}}(\rho)\right)_{n \geq 1} \quad\left(\operatorname{resp} . \quad \sigma_{t} \in\right.$ $\left.\operatorname{LIM}\left(i_{a^{n}}\left(\rho_{t}\right)\right)_{n \geq 1}\right)$ : Then we have

$$
A=C(k)+\sigma^{k}(A)\left(\text { resp. } A=C(t)+\sigma_{t}(A)\right) \text { etc. }
$$

『As above we obtain the representation

$$
A_{n}=\alpha^{-n} \cdot\left(a^{n}\left(\nu\left(\rho^{k}\right)-\varepsilon_{e}\right)+a^{n}\left(\nu\left(\rho^{k}\right)\right) * i_{a^{n}}\left(\rho^{k}\right)\left(A_{n}\right) .\right.
$$

Now we conclude as before: Assume $i_{a}^{n}(\rho) \stackrel{n \in\left(n^{\prime}\right)}{\longrightarrow} \sigma$. The second summands converge to $\sigma^{k}(A)$, hence the first converge too and we obtain $A=C(k)+$ $\sigma^{k}(A)$ for any accumulation point $\sigma \in \Lambda$ and some generating functional $C(k) \in \mathcal{G} \mathcal{F}(\mathbb{G})$. If $(* * *)$ holds true then $\sigma \in \rho \cdot \operatorname{Inv}(\mu)$, hence $\sigma$ may be replaced by $\rho$. $]$

b) $\sigma$ (resp. $\sigma_{t}: t \geq 0$ ) is contracting if $\rho$ (resp. $\rho_{t}: t \geq 0$ ) has this property.

IThis is easily proved representing $\rho$ (resp. $\left.i_{a}^{n}(\rho)\right)$ by the differentials $\rho^{o}$ (resp. $\left(a^{n} \rho a^{-n}\right)^{o}=a^{o} \rho^{o} a^{o}{ }^{-n}$ ) on the tangent space $\mathbb{V}$ (cf. Proposition 4.1 below):

$\rho$ is contracting iff we have $\operatorname{Spec}\left(a^{o} \rho^{o} a^{o}-n\right)=\operatorname{Spec}\left(\rho^{o}\right) \subset\{\alpha:|\alpha| \leq r\}$ for some $0<r<1$. I.e. the spectral radius is at most $r$ for all $n$. Hence $\operatorname{Spec}\left(\sigma^{o}\right) \subseteq\{\alpha:|\alpha| \leq r\}$ for all $\sigma \in \Lambda$. $]$

This is a motivation for further investigations: We want to show that the commutativity assumptions $(*)$ (resp. $(* *)$ and $(* * *)$ ) are no serious restrictions: They will turn out to be natural in special situations, e.g. if the measures under consideration are selfdecomposable (Section 4) and if the cofactors commute. (It is not hard to find examples of selfdecomposable laws with commuting cofactors, see e.g. [21]).

\section{Symmetric $\rho$-decomposable laws}

Here we consider a special situation: semistable strongly $\rho$-decomposable laws with symmetric cofactors. Therefore we assume the following conditions in this section:
(1) $\mu=\widetilde{\mu}$,
(2) $\mu=\nu(\rho) * \rho(\mu)$,
(3) $\rho(\mu) * \nu(\rho)=\nu(\rho) * \rho(\mu)$,
(4) $\widetilde{\nu(\rho)}=\nu(\rho)$ and
(5) $\rho$ is contractive. 
As usual, $\widetilde{\mu}$ will denote the image of $\mu \in \mathcal{M}^{1}(\mathbb{G})$ under the mapping $x \mapsto x^{-1}$ on $\mathbb{G}$. (Note that $(1),(2),(4) \Rightarrow(3)$ and that $\mu$ is strongly $\rho$-decomposable if (2) and (5) are fulfilled.)

We start with general results for symmetric convolution semigroups on root compact groups.

Lemma 3.1. a) Let $\mathbb{G}$ be a locally compact and 2-root compact group (see e.g. [8] for a definition). Let $\left(\lambda_{n}\right)_{n \geq 1} \subseteq \mathcal{M}^{1}(\mathbb{G})$. Assume (6) $\lambda_{n}=\chi_{n} * \nu_{n}=\nu_{n} * \chi_{n}$, furthermore $\widetilde{\chi}_{n}=\chi_{n}$ and $\nu_{n}=\widetilde{\nu}_{n}$, hence $\lambda_{n}=\widetilde{\lambda}_{n}$. Assume $\lambda_{n} \rightarrow \varepsilon_{e}$ and assume the existence of $k(n) \nearrow \infty$ such that $\lambda_{n}^{k(n)} \rightarrow \xi \in \mathcal{M}^{1}(\mathbb{G})$.

Then there exists a continuous convolution semigroup $\xi_{\bullet}$ with $\xi_{1}=\xi$, $\xi_{t}=\widetilde{\xi}_{t}$, with $\lambda_{n}^{[k(n) t]} \rightarrow \xi_{t}, t \geq 0$, furthermore $\left(\chi_{n}^{k(n)}\right)_{n \geq 1}$ and $\left(\nu_{n}^{k(n)}\right)_{n \geq 1}$ are relatively compact.

And for any accumulation point $(\kappa, \eta)=\lim _{n \in\left(n^{\prime}\right)}\left(\chi_{n}^{k(n)}, \eta_{n}^{k(n)}\right)$ there exist symmetric continuous convolution semigroups $\kappa_{\bullet}, \eta_{\bullet}$, such that

$$
(\kappa, \eta)=\left(\kappa_{1}, \eta_{1}\right) \text { and } \chi_{n}^{[k(n) t]} \rightarrow \kappa_{t}, \nu_{n}^{[k(n) t]} \rightarrow \eta_{t}, n \in\left(n^{\prime}\right),
$$

furthermore $\xi_{t}=\kappa_{t} * \eta_{t}$, all $t \geq 0$. And in addition $\left(\xi_{r}, \kappa_{s}, \eta_{t}\right)$ commute for all $r, s, t \geq 0$.

b) Let $\mathbb{G}$ be a locally compact group, let $\lambda_{\bullet}, \chi_{\bullet}^{\prime}, \chi_{\bullet}, \nu_{\bullet}$ be symmetric continuous convolution semigroups such that $\lambda_{t}=\chi_{t} * \nu_{t}=\chi_{t}^{\prime} * \nu_{t}, t \geq 0$, and $\lambda_{0}=\varepsilon_{e}$. Then $\chi_{\bullet} \equiv \chi_{\bullet}^{\prime}$.

Proof. a) 1. Let $\left(\sigma_{n}\right)_{n \geq 1} \subseteq \mathcal{M}^{1}(\mathbb{G})$ be symmetric and shift compact. Then $\left(\sigma_{n}\right)_{n \geq 1}$ is relatively compact. $\llbracket\left(\sigma_{n}^{2}=\sigma_{n} * \widetilde{\sigma}_{n}\right)_{n \geq 1}$ is relatively compact. Therefore the 2-root-compactness of $\mathbb{G}$ implies relative compactness of $\left(\sigma_{n}\right)_{n \geq 1}$. ]

2. We have $\lambda_{n}^{k(n)} \rightarrow \xi, \lambda_{n} \rightarrow \varepsilon_{e}$ and $\lambda_{n}^{k(n)}=\lambda_{n}^{2[k(n) / 2]} * \lambda_{n}^{\varepsilon(n)}$ with $\varepsilon(n) \in$ $\{0,1\}$, hence $\lambda_{n}^{\varepsilon(n)} \rightarrow \varepsilon_{e}$. 2-root-compactness of $\mathbb{G}$ implies therefore the existence of $\xi_{1 / 2} \in \mathcal{M}^{1}(\mathbb{G})$ such that $\lambda_{n}^{[k(n) / 2]} \rightarrow \xi_{1 / 2}$. And successively we obtain $\lambda_{n}^{[k(n) t]} \rightarrow \xi_{t}, t \in \mathbb{D}_{+}$, where $\mathbb{D}$ denotes the dyadic numbers. And $\mathbb{D}_{+} \ni t \mapsto \xi_{t}$ is a $*$-homomorphism.

3. Observing $\lambda_{n}^{[k(n) t]} \rightarrow \xi_{t}$ and $\lambda_{n}^{[k(n) t]}=\chi_{n}^{[k(n) t]} * \nu_{n}^{[k(n) t]}$ (by assumption (6)), we obtain that the sequences of factors are symmetric and shift compact. Therefore according to step 1 , we conclude that $\left(\chi_{n}^{[k(n) t]}\right)_{n \geq 1}$ and $\left(\nu_{n}^{[k(n) t]}\right)_{n \geq 1}$ are relatively compact for any $t \in \mathbb{D}_{+}$.

Fix a subsequence $\left(n^{\prime}\right)$ such that $\left(\kappa_{1}, \eta_{1}\right)=\lim _{n \in\left(n^{\prime}\right)}\left(\chi_{n}^{k(n)}, \nu_{n}^{k(n)}\right)$. Repeating the arguments of step 2 we conclude $\chi_{n}^{[k(n) t] \stackrel{n \in\left(n^{\prime}\right)}{\longrightarrow}} \kappa_{t}$ and 


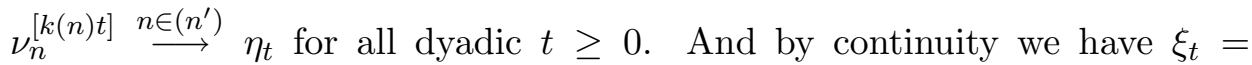
$\kappa_{t} * \eta_{t}=\eta_{t} * \kappa_{t}$ and $\xi_{t}, \kappa_{t}, \eta_{t}$ are symmetric.

4. But $\kappa_{t}=\kappa_{t / 2} * \widetilde{\kappa}_{t / 2}, \xi_{t}=\xi_{t / 2} * \widetilde{\xi}_{t / 2}$ and $\eta_{t}=\eta_{t / 2} * \widetilde{\eta}_{t / 2}, t \in \mathbb{D}_{+}$, hence the corresponding convolution operators on $L^{2}(\mathbb{G})$ are positive semidefinite. Let $T_{t}, S_{t}, R_{t}$ denote the convolution operators corresponding to $\xi_{t}, \kappa_{t}$ and $\eta_{t}$ on $L^{2}(\mathbb{G})$. Uniqueness of positive semidefinite roots of positive semidefinite operators finally implies that $\xi_{t}, \kappa_{t}$ and $\eta_{t}$ are uniquely determined for $t \in \mathbb{D}_{+}$.

Defining $T_{t}:=T^{t}$ by the spectral resolution for all $t \geq 0$ we obtain an uniquely determined extension $\left(T_{t}\right)_{t>0}$. And this contraction semigroup is continuous w.r.t. the strong operator topology. Comparing the weak operator topology on the set of convolution operators and the vague topology on the compact set $\left\{\lambda \in \mathcal{M}_{+}(\mathbb{G}):\|\lambda\| \leq 1\right\}$ we conclude the existence of measures $\xi_{t}$ such that $T_{t}$ is representable as convolution operator for all real $t \geq 0$. $\xi_{\bullet}$ is a vaguely continuous convolution semigroup such that $\xi_{t} \in \mathcal{M}^{1}(\mathbb{G})$ for $t \in \mathbb{D}_{+}$. Whence $\left(\xi_{t}\right)_{t \geq 0} \subseteq \mathcal{M}^{1}(\mathbb{G})$ and weak continuity easily follow.

Analogous representations exist for $S_{t}$ and $R_{t}$. Hence we obtain extensions to continuous convolution semigroups $\left(\xi_{t}\right)_{t \in \mathbb{R}_{+}},\left(\kappa_{t}\right)_{t \in \mathbb{R}_{+}}$and $\left(\eta_{t}\right)_{t \in \mathbb{R}_{+}}$ and we have $\lambda_{n}^{[k(n) t]} \rightarrow \xi_{t}, \chi_{n}^{[k(n) t]} \rightarrow \kappa_{t}, \nu_{n}^{[k(n) t]} \rightarrow \eta_{t}, n \in\left(n^{\prime}\right)$, for all $t \geq 0$. 5. We have: $T_{t}=S_{t} R_{t}=R_{t} S_{t}$, and $T_{t}, S_{t}, R_{t} \geq 0$. $T_{t}, S_{t}$ and $R_{t}$ are functions of $T_{1}, S_{1}$ and $R_{1}$ and hence $T_{t}, S_{t}$ and $R_{t}$ belong to the double commutator of $T_{1}, S_{1}$ and $R_{1}$ respectively. Whence $S_{r} R_{t}=R_{t} S_{r}$ for all $r, t \geq 0$, and the assertion follows.

b) The assumptions yield $\chi_{0}=\chi_{0}^{\prime}=\varepsilon_{e}$. For symmetric continuous convolution semigroups the convolution operators are injective (cf. [22], Lemma 1). Whence the assertion follows.

For the next result we need a convergence of types theorem. Therefore we assume again $\mathbb{G}$ to be a simply connected nilpotent Lie group (see e.g. $[5],[2])$.

Proposition 3.2. Assume the properties (1)-(5) and assume in addition that $\mu=\mu_{1}$ is embeddable into a symmetric continuous convolution semigroup $\mu_{\bullet}$ which is $(a, \alpha)$-semistable. Let $\mu$ be full.

a) Then $\Lambda:=\left\{i_{a}^{n}(\rho):=a^{n} \rho a^{-n}: n \in \mathbb{N}\right\}$ is relatively compact in $\mathrm{C}(\mu)$ and any $\sigma \in \operatorname{LIM}(\Lambda)$ is contracting and fulfils $\mu_{t}=\kappa_{t}^{\sigma} * \sigma\left(\mu_{t}\right)$, all $t \geq$ 0, i.e. $\sigma \in \mathrm{C}\left(\mu_{t}\right)$ and $\kappa_{t}^{\sigma} \in \operatorname{Cof}_{\mu_{t}}(\sigma), t \geq 0$. Here $\kappa_{\bullet}^{\sigma}=\left(\kappa_{t}^{\sigma}\right)_{t \geq 0}$ is a symmetric continuous $(a, \alpha)$-semistable convolution semigroup. Moreover, $\left(\mu_{r}, \kappa_{s}^{\sigma}, \sigma\left(\mu_{t}\right)\right)$ commute for all $r, s, t \geq 0$. In particular, $\sigma \in \mathrm{C}\left(\mu_{t}\right), t \geq 0$.

b) If $A$ and $B^{\sigma}$ denote the generating functionals of $\mu_{\bullet}$ and $\kappa_{\bullet}^{\sigma}$ respectively then we have $A=B^{\sigma}+\sigma(A)$. 
c) If $\operatorname{LIM}(\Lambda)$ is uniquely determined $\bmod \operatorname{Inv}(\mu)$, i.e. if $i_{a}^{n}(\rho) \cdot \operatorname{Inv}(\mu)$ $\stackrel{n \rightarrow \infty}{\longrightarrow} \sigma \cdot \operatorname{Inv}(\mu)$, then $\kappa_{\bullet}^{\sigma}=\kappa_{\bullet}$ is uniquely determined and $\mu_{t}=\kappa_{t} * \sigma\left(\mu_{t}\right)$ follows. Furthermore, we have (*) $i_{a}(\sigma) \in \sigma \cdot \operatorname{Inv}(\mu)$. (Note that $\operatorname{Inv}(\mu)=\operatorname{Inv}\left(\mu_{t}\right), t>0$, cf. Remark in Section 2.)

d) In particular, if $i_{a}(\rho) \in \rho \operatorname{Inv}(\mu)$ we may put $\sigma=\rho$ and $\kappa_{1}=\nu(\rho)$.

Proof. a) Put $k(n):=\left[\alpha^{-n}\right]$. Then we have $a^{n} \mu_{1}^{[k(n) t]} \rightarrow \mu_{t}, t \geq 0$. Furthermore, $\mu_{1}=\nu(\rho) * \rho\left(\mu_{1}\right)$, hence $\mu_{1}^{n}=\nu(\rho)^{n} * \rho\left(\mu_{1}\right)^{n}, n \in \mathbb{N}$, (according to the commutativity assumption). Therefore Lemma 3.1 a) applies with $\lambda_{n}:=a^{n}\left(\mu_{1}\right), \chi_{n}:=a^{n}(\nu(\rho))$ and $\nu_{n}:=a^{n} \rho\left(\mu_{1}\right)$.

In particular, $\left\{\nu_{n}^{k(n)}=a^{n} \rho a^{-n}\left(\mu_{\alpha^{n}}^{k(n)}\right)=i_{a}^{n}(\rho)\left(\mu_{\alpha^{n}}^{k(n)}\right)\right\}$ is relatively compact. Since $\mu_{\alpha^{n}}^{k(n)} \rightarrow \mu_{1}$, according to the convergence of types theorem the sequence $\Lambda=\left\{i_{a}^{n}(\rho)\right\}$ is relatively compact and any limit point of $\left\{\nu_{n}^{k(n)}\right\}$ is of the form $\sigma\left(\mu_{1}\right)$ for some $\sigma \in \operatorname{LIM}(\Lambda)$.

Fix a subsequence $\left(n^{\prime}\right)$ such that $\left(\nu_{n}^{k(n)}, i_{a}^{n}(\rho)\right) \stackrel{n \in\left(n^{\prime}\right)}{\longrightarrow}\left(\sigma\left(\mu_{1}\right)=: \eta_{1}^{\sigma}, \sigma\right)$. According to Lemma 3.1 a) there exist embedding continuous convolution semigroups $\kappa_{\bullet}^{\sigma}$ and $\eta_{\bullet}^{\sigma}$ such that $\mu_{t}=\kappa_{t}^{\sigma} * \eta_{t}^{\sigma}$ and $\kappa_{s}^{\sigma} * \eta_{t}^{\sigma}=\eta_{t}^{\sigma} * \kappa_{s}^{\sigma}, t, s \geq 0$. And furthermore $a^{n} \rho\left(\mu_{1}\right)^{[k(n) t]} \rightarrow \eta_{t}^{\sigma}$ and $a^{n}(\nu(\rho))^{[k(n) t]} \rightarrow \kappa_{t}^{\sigma}, n \in\left(n^{\prime}\right)$, for all $t \geq 0$.

On the other hand, we have for $t \geq 0$ : $i_{a}^{n}(\rho)\left(\mu_{t}\right)=a^{n} \rho a^{-n}\left(\mu_{t}\right)=$ $a^{n} \rho\left(\mu_{\alpha^{-n} t}\right)=a^{n} \rho\left(\mu_{[k(n) t]+s_{n}(t)}\right)=a^{n} \rho\left(\mu^{[k(n) t]}\right) * a^{n} \rho\left(\mu_{s_{n}(t)}\right)\left(\right.$ with $s_{n}(t) \rightarrow$ $0)$; hence - since $a$ is contracting $-i_{a}^{n}(\rho)\left(\mu_{t}\right) \rightarrow \eta_{t}^{\sigma}$, and therefore by continuity, $\eta_{\bullet}^{\sigma}=\sigma\left(\mu_{\bullet}\right)$.

b) follows immediately since $a$ is contracting.

c) In particular if $\left\{i_{a}^{n}(\rho) \cdot \operatorname{Inv}(\mu)\right\}_{n \geq 1}$ converges to $\sigma \cdot \operatorname{Inv}(\mu)$, then we conclude that $\sigma\left(\mu_{\bullet}\right)$ - and hence by Lemma $\left.3.1 \mathrm{~b}\right)$ also $\kappa_{\bullet}^{\sigma}=\kappa_{\bullet}$ - are uniquely determined, therefore, $i_{a}(\sigma)\left(\mu_{t}\right)=\sigma\left(\mu_{t}\right)=\eta_{t}$, all $t \geq 0$. Whence $(*) \quad i_{a}(\sigma) \in \sigma \operatorname{Inv}\left(\mu_{t}\right)$.

Furthermore, $a^{n} \nu(\rho) \stackrel{[k(n) t]}{\stackrel{n \rightarrow \infty}{\longrightarrow}} \kappa_{t}$, and we conclude that $\nu(\rho)$ belongs to the normal domain of semistable attraction of $\kappa_{\bullet}$. Therefore $\kappa_{\bullet}$ is $(a, \alpha)$ semistable (cf. [18]).

d) If $a \rho a^{-1} \in \rho \cdot \operatorname{Inv}\left(\mu_{t}\right)$ for all $t>0$, then $\sigma \in \rho \cdot \operatorname{Inv}\left(\mu_{t}\right)=\rho \cdot \operatorname{Inv}\left(\mu_{1}\right)$ for $t>0$.

It rests to show contractivity of $\sigma$ : This is done almost verbatim as in the proof of $2.3 \mathrm{~b}$ ).

In a similar way we obtain - again for a simply connected nilpotent Lie group - that instead of semistable laws we may consider probabilities belonging to the domain of semistable attraction: 
Proposition 3.3. Let $\mu \in \mathcal{M}^{1}(\mathbb{G})$ be $\rho$-decomposable with contracting $\rho \in$ Aut $(\mathbb{G})$ and cofactor $\nu(\rho)$. Assume the properties (1)-(4). Assume the existence of $k(n) \nearrow \infty$ and $a_{n} \in \operatorname{Aut}(\mathbb{G})$ such that $a_{n}\left(\mu^{k(n)}\right) \rightarrow \xi \in \mathcal{M}^{1}(\mathbb{G})$, a full measure.

a) Then $\xi$ is embeddable into a symmetric continuous convolution semigroup $\xi_{\bullet}$ with $\xi_{1}=\xi$, and we have: $\Lambda:=\left\{i_{a_{n}}(\rho): n \geq 1\right\}$ is relatively compact and for any $\sigma \in \operatorname{LIM}(\Lambda)$ there exists a symmetric continuous convolution semigroup $\kappa_{\bullet}^{\sigma}$ such that $\xi_{t}=\kappa_{t}^{\sigma} * \sigma\left(\xi_{t}\right), t \geq 0$. In addition, $\left(\xi_{r}, \kappa_{s}^{\sigma}, \sigma\left(\xi_{t}\right)\right)$, commute for $r, s, t \geq 0$. In particular, $\sigma \in C\left(\xi_{t}\right), t \geq 0$.

b) If in addition $k(n) / k(n+1) \rightarrow \alpha \in(0,1)$ then $\xi_{\bullet}$ is $(a, \alpha)$-semistable for some $a \in \operatorname{LIM}\left(a_{n} a_{n+1}^{-1}\right)$. And if in addition $i_{a}^{n}(\rho) \cdot \operatorname{Inv}(\xi) \stackrel{n \rightarrow \infty}{\longrightarrow} \sigma \cdot \operatorname{Inv}(\xi)$, then $\kappa_{\bullet}^{\sigma}=\kappa_{\bullet}$ is uniquely determined and furthermore $\kappa_{\bullet}$ is $(a, \alpha)$-semistable. Moreover, we have $(*) \quad i_{a}(\sigma) \in \sigma \operatorname{Inv}\left(\xi_{t}\right), t \geq 0$.

c) If $i_{a_{n}}(\rho) \in \rho \operatorname{Inv}\left(\xi_{t}\right)$ then $\sigma \in \rho \cdot \operatorname{Inv}\left(\xi_{t}\right)$, hence we may put $\sigma=\rho$.

Proof. Analogous to the proof of Proposition 3.2: Apply Lemma 3.1 a) to $\lambda_{n}:=a_{n}(\mu), \chi_{n}:=a_{n}(\nu(\rho)), \nu_{n}:=a_{n} \rho(\mu)$. Therefore $a_{n}(\mu)^{k(n)} \rightarrow \xi$ yields the embeddability of $\xi$ into $\xi_{\bullet}, a_{n}(\mu)^{[k(n) t]} \rightarrow \xi_{t}$, as well as relative compactness of $\left\{a_{n} \rho(\mu)^{[k(n) t]}=i_{a_{n}}(\rho) a_{n}(\mu)^{[k(n) t]}\right\},\left\{a_{n}(\nu(\rho))^{[k(n) t]}\right\}$ and of $\left\{i_{a}^{n}(\rho)\right\}$ (by the convergence of types theorem).

For any subsequence $\left(n^{\prime}\right)$ with $i_{a}^{n}(\rho) \rightarrow \sigma \psi, \psi \in \operatorname{Inv}(\xi)$, we obtain:

$$
i_{a_{n}}(\rho) a_{n}(\mu)^{[k(n) t]} \rightarrow \sigma\left(\mu_{t}\right), \quad a_{n}(\nu(\rho))^{[k(n) t]} \rightarrow \kappa_{t}^{\sigma}, n \in\left(n^{\prime}\right),
$$

and $\xi_{t}=\kappa_{t}^{\sigma} * \sigma\left(\mu_{t}\right)=\sigma\left(\mu_{t}\right) * \kappa_{t}^{\sigma}$, for all $t \geq 0$.

We continue as in the proof of 3.2: Contractivity of $\sigma$ is proved analogously to Proposition 3.2 resp. $2.3 \mathrm{~b}$ ); If $\sigma\left(\mu_{\bullet}\right)$ is uniquely determined, then by $3.1 \mathrm{~b}$ ) also $\kappa_{\bullet}^{\sigma}=\kappa_{\bullet}$ is uniquely determined and (*) follows as in 3.2.

Semistability of $\xi_{\bullet}$ follows observing that $\xi_{\bullet}$ is full and the domain of semistable attraction of $\xi_{\bullet}$ is non-void. Hence $\xi_{\bullet}$ is $(a, \alpha)$-semistable for some $a \in \operatorname{LIM}\left(a_{n} a_{n+1}^{-1}\right)$ (cf. [18]). Therefore the generating functionals $A$ and $B$ of $\xi_{\bullet}$ and $\kappa_{\bullet}$ fulfil the relation $A=B+\sigma(A)$ and by (*) also $\alpha \cdot A=a(B)+\alpha \cdot \sigma(A)$ follows. Semistability of $\kappa_{\bullet}$ follows now analogously to the proof of Theorem $2.2 \mathrm{a}$ ).

\section{Semistable selfdecomposable laws}

Let $\mathbb{G}$ be a simply connected nilpotent Lie group with Lie algebra $\mathbb{V}$. We are now motivated to study semistable selfdecomposable laws with commuting cofactors. Our aim is to show that the commutativity assumption

$$
a \rho_{t}=\rho_{t} a, \quad t \geq 0,
$$

is no serious restriction in this situation. 
We follow the investigations of A. Luczak [17] for vector spaces (continuing R. Shah's investigations into selfdecomposability on groups [21], [1]) to show finally that A. Łuczak's characterization may be applied to obtain a description of full semistable selfdecomposable laws on simply connected nilpotent Lie groups.

We start with preparatory facts and notations which are well known and folklore or are proved almost verbatim as for vector spaces.

Proposition 4.1. 1. $\mathbb{G}$ is simply connected, hence Aut $(\mathbb{G})$ is isomorphic to $\operatorname{Aut}(\mathbb{V}) \subseteq \mathrm{GL}(\mathbb{V})$, the group of Lie algebra automorphisms of $\mathbb{V}$; analogously $\operatorname{End}(\mathbb{G})$ is isomorphic to $\mathrm{E}(\mathbb{V}):=\{A \in \operatorname{End}(\mathbb{V}): A[x, y]=[A x, A y]$ for $x, y \in \mathbb{V}\}$, the semigroup of Lie algebra endomorphisms. The isomorphism is denoted by

$$
\begin{gathered}
a \in \operatorname{End}(\mathbb{G}) \leftrightarrow a^{o} \in \mathrm{E}(\mathbb{V}), a^{o} \text { denoting the differential of } a \text {, defined by } \\
\qquad \exp a^{o}(x)=a(\exp (x)), x \in \mathbb{V} .
\end{gathered}
$$

〔For details concerning Lie groups, Lie algebras and endomorphisms the reader is referred to [9], in particular VII, 3.3, 3.4, 4.2 and IX, 1.2. Concerning the isomorphism Aut $(\mathbb{G}) \cong \operatorname{Aut}(\mathbb{V})$ for simply connected Lie groups see especially [9], XII, remark on p. 134.]

2. Let $\mu \in \mathcal{M}^{1}(\mathbb{G})$ be $S$-full (i.e. not concentrated on a coset of a proper closed connected subgroup). Then $\mathrm{D}(\mu)$ (as well as $\mathrm{D}^{r}(\mu), \mathrm{D}^{c}(\mu)$ and $\mathrm{DC}(\mu)$ ) are compact semigroups in $\operatorname{End}(\mathbb{G})$ and $\mathrm{C}(\mu)$ is a compact subset $\subseteq \mathrm{DC}(\mu)$. Via the exponential mapping we obtain that

$$
\begin{aligned}
& \mathrm{D}(\mu)^{o}:=\left\{a^{o}: a \in \mathrm{D}(\mu)\right\}=: \Delta(\mu) \\
& \text { is a compact subsemigroup } \subseteq \mathrm{E}(\mathbb{V}) \subseteq \operatorname{End}(\mathbb{V}) .
\end{aligned}
$$

In this way the analysis of the topological semigroup $\mathrm{D}(\mu)$ is reduced to the analysis of a concrete (matrix-) semigroup $\Delta(\mu)$. Analogously, $\mathrm{D}^{r}(\mu)^{o}=$ $\Delta^{r}(\mu), \mathrm{D}^{c}(\mu)^{o}=\Delta^{c}(\mu)$ and $\mathrm{DC}(\mu)^{o}=: \Gamma(\mu)$ are treated as subsemigroups of $\operatorname{End}(\mathbb{V})$. For typographical reasons we shall concentrate on $\mathrm{D}(\mu)$ and $\Delta(\mu)$ in the following.

【 As in the vector space case, it is immediately seen that $\mathrm{D}, \mathrm{D}^{c}, \mathrm{DC}$ are semigroups. The compactness-assertions are immediate consequences of the convergence of types theorem, cf. e.g. [5]; see also [21], Lemma 3. ]

3. Let $\mathcal{A}(\mu)$ denote the maximal subgroup of $\mathrm{D}(\mu)$ and let $\mathcal{A}^{o}(\mu)$ be the corresponding object in $\Delta(\mu) \subseteq \mathrm{E}(\mathbb{V})$.

Furthermore, in analogy to vector spaces we define $\mathrm{E}(\mu):=\{Q \in \operatorname{End}(\mathbb{V})$ such that $e^{t Q} \in \operatorname{Aut}(\mathbb{V}), t \in \mathbb{R}$ and $e^{t Q} \in \Delta(\mu)$ for all $\left.t \geq 0\right\}$. Hence $Q \in \mathrm{E}(\mu)$ iff there exists a one-parameter group $\left(\rho_{t}\right)_{t \in \mathbb{R}} \subseteq \operatorname{Aut}(\mathbb{G})$ such that $\rho_{t}^{o}=e^{t Q}$ and $\left\{\rho_{t}: t \geq 0\right\} \subseteq \mathrm{D}(\mu) \llbracket$ hence $\rho_{t}^{o} \in \Delta(\mu) \rrbracket$. In particular, $Q$ 
belongs to $\operatorname{Der}(\mathbb{V})(\cong \operatorname{Der}(\mathbb{G}))$, the derivations of the Lie algebra $\mathbb{V}$ (resp. the Lie group $\mathbb{G})$.

Furthermore, we consider the subsets $\mathrm{E}^{0}(\mu):=\left\{Q \in \mathrm{E}(\mu): e^{t Q} \in\right.$ $\left.\mathcal{A}^{o}(\mu), t \geq 0\right\}$ and $\mathrm{E}^{-}(\mu):=\left\{Q \in \mathrm{E}(\mu):\left(\rho_{t}\right)^{o}=\left(e^{t Q}\right)_{t \geq 0}\right.$ is contractive on $\mathbb{V}\}=\left\{Q \in \mathrm{E}(\mu):\left(\rho_{t}\right)_{t \geq 0}\right.$ is contractive on $\left.\mathbb{G}\right\}$. I.e. $Q \in \mathrm{E}^{-}(\mu)$ iff $Q \in \mathrm{E}(\mu)$ and $\operatorname{Spec}(Q) \subseteq\{\alpha \in \mathbb{C}: \Re \alpha<0\}$ and $Q \in \mathrm{E}^{0}(\mu)$ iff $Q \in \mathrm{E}(\mu)$ and $\operatorname{Spec}(Q) \subseteq\{\alpha \in \mathbb{C}: \Re \alpha=0\}$. [Cf. [17] ]

The following elementary observations are folklore:

4. Let $A, A_{n}, Q_{n}, S_{n}$ be linear operators on a vector space $\mathbb{V}=\mathbb{R}^{d}$. Let $r(\cdot)$ denote the spectral radius. Then

a) If $A_{n} \rightarrow A$ then $r(A) \leq \liminf r\left(A_{n}\right)$.

b) Hence, if $Q_{k} \in E^{-}(\mu)$ fulfilling the relation $\operatorname{Spec}\left(Q_{k}\right) \subseteq\{\Re \alpha \leq-\varepsilon\}$ for some $\varepsilon>0$ and $Q_{k} \rightarrow Q$ then $\operatorname{Spec}(Q)$ fulfils the same relation, in particular, $Q \in E^{-}(\mu)$.

5. Mean ergodic theorem for finite dimensional vector spaces $\mathbb{W}:$ Let $T \in \operatorname{End}(\mathbb{W})$ and $x \in \mathbb{W}$ be such that the orbit $\left\{T^{n}(x): n \geq 0\right\}$ is bounded in $\mathbb{W}$.

a) Then the $\mathrm{C}-1$-means $T(n)(x):=(1 / n) \sum_{1}^{n} T^{k}(x)$ converge to a fixpoint $y=T(y)$.

b) Furthermore, for any accumulation point $w=\lim _{j \geq 1} T^{n_{j}}(x)$ of the orbit we have $T(n)(w)=(1 / n) \sum_{1}^{n} T^{k}(w) \rightarrow y$. In particular, $y \in$ $\overline{\mathrm{CO}}\left(\operatorname{LIM}\left\{T^{n}(x): n \geq 0\right\}\right)$. (Note that $T(n)$ are convex combinations of $T^{k}$.)

See e.g. [12], Chapter 2, 1.3 and 2.1: $\mathbb{W}$ is finite dimensional, hence w.l.o.g. we may assume $T$ to be powerbounded on $\mathbb{W}$ and hence $\{\|T(n)\|: n \geq 1\}$ to be bounded. Then we have a decomposition $\mathbb{W}=\mathrm{F} \oplus \mathrm{N}$, where $\mathrm{F}$ is the fixpoint space of $T$ - therefore $\left.T(n)\right|_{\mathrm{F}}=\left.\mathrm{id}\right|_{\mathrm{F}}-$ and $T(n) \rightarrow 0$ on N. Now the assertion follows immediately observing that $T(n) T^{n_{j}}(x) \stackrel{n \rightarrow \infty}{\longrightarrow} T^{n_{j}}(y)=$ $y$ for all $j$ and hence $T(n)(w) \rightarrow y$.

6. Let $\mu_{n}, \mu$ be $S$-full measures with $\mu_{n} \rightarrow \mu$. Then $\bigcup_{n} \mathrm{D}\left(\mu_{n}\right)$ is relatively compact with $\operatorname{LIM}\left(\bigcup_{n \geq 1} \mathrm{D}\left(\mu_{n}\right)\right) \subseteq \mathrm{D}(\mu)$. In fact, if $\mu_{n}=\gamma_{n} * b_{n}\left(\mu_{n}\right), n \in \mathbb{N}$, then $\left\{b_{n}\right\}$ and $\left\{\gamma_{n}\right\}$ are relatively compact and for any accumulation point $(b, \gamma)$ we have $\mu=\gamma * b(\mu)$.

IImmediate consequence of the convergence of types theorem for endomorphisms [5]: By factor compactness (cf. [19]) there exists $\left\{x_{n}\right\} \subseteq \mathbb{G}$ such that $\left\{\gamma_{n} * \varepsilon_{x_{n}}\right\}$ and $\left\{\varepsilon_{x_{n}^{-1}} * b_{n}\left(\mu_{n}\right)\right\}$ are relatively compact. The convergence of types theorem yields relative compactness of $\left\{\left(b_{n}, x_{n}\right)\right\}$ in $\operatorname{End}(\mathbb{G}) \times \mathbb{G}$. Hence in particular $\left\{x_{n}\right\}$ is relatively compact and therefore $\left\{b_{n}\left(\mu_{n}\right)\right\}$ and $\left\{\gamma_{n}\right\}$ are relatively compact. Whence the assertion. ] 
Analogous results hold true for $\mathrm{D}^{r}(\mu), \mathrm{D}^{c}(\mu), \mathrm{DC}(\mu)$ and $C(\mu)$.

7. $\mathrm{E}(\mu)$ and $\mathrm{E}^{-}(\mu)$ are convex cones in $\operatorname{End}(\mathbb{V}), \mathrm{E}^{0}(\mu)$ is a linear subspace; $\mathrm{E}(\mu)$ is closed in $\operatorname{End}(\mathbb{V})$. Furthermore,

$$
Q_{i} \in \mathrm{E}(\mu) \backslash \mathrm{E}^{0}(\mu), i=1,2 \Rightarrow Q_{1}+Q_{2} \in \mathrm{E}(\mu) \backslash \mathrm{E}^{0}(\mu) .
$$

«Almost verbatim as [17], Lemma 2 and Corollary 3. ॥

8. Applying 6 we obtain: Let $\mu_{j} \rightarrow \mu$, a S-full measure, and let $Q_{j} \in$ $\mathrm{E}\left(\mu_{j}\right)$ such that $Q_{j} \rightarrow Q \in \operatorname{End}(\mathbb{V})$. Then $Q \in \mathrm{E}(\mu)$.

[ Let $\left(a_{t}^{(j)}\right)_{t \geq 0} \subseteq \mathrm{D}\left(\mu_{j}\right)$ be defined by $\left(a_{t}^{(j)}\right)^{o}=e^{t Q_{j}}, j \geq 1, t \geq 0$. Then by assumption $a_{t}:=\lim _{j \geq 0} a_{t}^{(j)}$ exists with $a_{t}^{o}=e^{t Q}, t \geq 0$, and belongs to $\mathrm{D}(\mu)$ according to step 6 . Hence $Q \in \mathrm{E}(\mu)$ as asserted.

Proposition 4.2. Let $\mu \in \mathcal{M}^{1}(\mathbb{G})$ be full and $(a, \alpha)$-semistable, let $\mu_{\bullet}$ denote the semistable convolution semigroup with $\mu_{1}=\mu$. Furthermore assume that $\left(\rho_{t}\right)_{t \in \mathbb{R}}$ is contracting and $\left(\rho_{t}\right)_{t \geq 0} \subseteq \mathrm{DC}(\mu)$, i.e. $\mu^{n}=\nu(t, n) *$ $\rho_{t}\left(\mu^{n}\right)=\rho_{t}\left(\mu^{n}\right) * \nu^{\prime}(t, n), t \geq 0, n \in \mathbb{N}$.

Then there exists a group $\left(\sigma_{t}\right)_{t \in \mathbb{R}}$, with $\sigma_{t}^{o}=e^{t Q}, Q \in E\left(\mu_{s}\right) \backslash E^{0}\left(\mu_{s}\right)$, such that

$$
\left(\sigma_{t}\right)_{t \geq 0} \subseteq \mathrm{DC}\left(\mu_{s}\right), \text { for all } s \geq 0, \text { and } \quad\left(*^{\prime}\right) \quad a \sigma_{t}=\sigma_{t} a \text { for all } t \geq 0 .
$$

Proof. 1. Put $k(n)=\left[\alpha^{-n}\right]$. Then for $s \geq 0$ we have $a^{n}\left(\mu^{[k(n) s]}\right) \rightarrow \mu_{s}$ and we obtain

$$
\begin{aligned}
a^{n}\left(\mu^{[k(n) s]}\right) & =a^{n}(\nu([(n) s], t)) * a^{n} \rho_{t}\left(\mu^{[k(n) s]}\right) \\
& =a^{n}(\nu([k(n) s], t)) * a^{n} \rho_{t} a^{-n}\left(a^{n} \mu^{[k(n) s]}\right),
\end{aligned}
$$

and an analogous decomposition for right cofactors since $\rho_{t} \in \mathrm{DC}(\mu)$.

Hence immediately $i_{a}^{n}\left(\rho_{t}\right) \subseteq \mathrm{DC}\left(\mu_{\alpha^{n}[k(n) s]}\right)$ for all $n$. According to 4.1 step 6, $\left\{a^{n} \rho_{t} a^{-n}\right\}_{n \geq 0}$ and $\left\{a^{n} \nu([k(n) s], t)\right\}_{n \geq 0}$ are relatively compact for any $s, t>0$. And for all accumulation points $(b(t), \gamma(s, t)) \in \operatorname{End}(\mathbb{G}) \times \mathcal{M}^{1}(\mathbb{G})$ we have $\mu_{s}=\gamma(s, t) * b(t)\left(\mu_{s}\right)=b(t)\left(\mu_{s}\right) * \gamma^{\prime}(s, t)$. Hence $b(t) \in \mathrm{DC}\left(\mu_{s}\right)$. (Indeed, one can choose $b(t)$ in such a way that $(b(t))_{t \geq 0}$ is a continuous semigroup belonging to $\mathrm{DC}\left(\mu_{s}\right)$.)

Let $\rho_{t}^{o}=: R_{t}=e^{t Q}, Q \in \mathrm{E}^{-}(\mu)$ and put $D:=a^{o}$. Then $\left(a^{n} \rho_{t} a^{-n}\right)^{o}=$ : $R_{t}^{(n)}=e^{t D^{n} Q D^{-n}}=$ : exp $t i_{D}^{n}(Q)$. Put $S_{n}:=i_{D}^{n}(Q)$ and $Q_{n}:=(1 / n)$ $\sum_{1}^{n} i_{D}^{k}(Q)$, let $s>0$.

2. $S_{n}=i_{D}^{n}(Q) \in \mathrm{E}^{-}\left(\mu_{\alpha^{n}[k(n) s]}\right)$ for all $n$, and for any accumulation point $S$ of $\left(S_{n}\right)$ we have $S \in \mathrm{E}\left(\mu_{s}\right) \backslash \mathrm{E}^{0}\left(\mu_{s}\right)$. (In fact, $S \in \mathrm{E}^{-}\left(\mu_{s}\right)$.) 
【 We have $\mu_{\alpha^{n}[k(n) s]}=a^{n}\left(\mu^{[k(n) s]}\right)=a^{n}\left(\nu([k(n) s], t) *\left(a^{n} \rho_{t} a^{-n}\right) a^{n}\left(\mu^{[k(n) s]}\right)=\right.$ $a^{n}\left(\nu([k(n) s], t) * \exp t S_{n}\left(\mu_{\alpha^{n}[k(n) s]}\right)\right.$, for all $n$ and $s, t \geq 0$. Assume $S_{n} \stackrel{n \in\left(n^{\prime}\right)}{\longrightarrow}$ $S$ for some subsequence $\left(n^{\prime}\right)$. The second assertion follows since $\operatorname{Spec} S_{n}=$ Spec $Q$, observing 4.1 , step 8 .

3. $\left\{S_{n}\right\}_{n \in \mathbb{N}}$ is bounded.

| Put e.g. $s=1$. Assume $\left\|S_{n_{j}}\right\| \rightarrow \infty$. Put $c_{j}:=\left\|S_{n_{j}}\right\|^{-1}$. Then $\bar{S}_{n_{j}}:=$ $c_{j} \cdot S_{n_{j}} \in \mathrm{E}^{-}\left(\mu_{\alpha^{n_{j}} k\left(n_{j}\right)}\right)$ (since $S_{n_{j}} \in \mathrm{E}^{-}\left(\mu_{\alpha^{n_{j}} k\left(n_{j}\right)}\right)$ according to 1), and

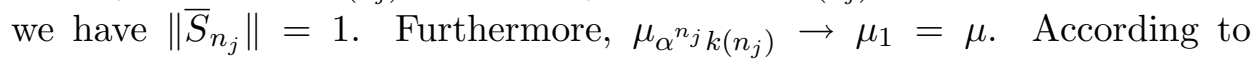
4.1 , step 6 and step 1 the sequence $\left\{e^{t \bar{S}_{n_{j}}}\right\}$ is relatively compact and all its accumulation points belong to $\Delta(\mu)$ for all $t \geq 0$. Now $\operatorname{Spec}\left(\bar{S}_{n_{j}}\right)=$ $c_{j} \cdot \operatorname{Spec}(Q)$ hence $\operatorname{Spec}(\bar{S})=\{0\}$ for any accumulation point $\bar{S}$ of $\left\{\bar{S}_{n_{j}}\right\}$. But $\bar{S} \in \mathrm{E}(\mu)$ (4.1 step 8) and hence $\left\{e^{t \bar{S}}\right\} \subseteq \mathcal{A}^{o}$, the maximal subgroup of $\Delta(\mu) . \mathcal{A}^{o}$ being compact and $\operatorname{Spec}(\bar{S})=\{0\}$ yields $\bar{S}=0$, a contradiction as $\|\bar{S}\|=\lim \left\|\bar{S}_{n_{j}}\right\|=1$. $\|$

4. Now $\left\{S_{n}=i_{D^{n}}(Q)=D^{n} Q D^{-n}\right\}_{n \geq 1}$ is bounded. Then the mean ergodic theorem $(4.1$, step 5$)$ applied to $\mathbb{W}:=\operatorname{End}(\mathbb{V})$ and $T:=i_{D}$ yields:

$$
\begin{aligned}
& \frac{1}{n} \sum_{1}^{n} S_{n} \rightarrow \widetilde{Q} \in \mathrm{E}(\mathbb{V}) \text { for some } \widetilde{Q} \in \operatorname{End}(\mathbb{V}) \\
& \text { such that } i_{D}(\widetilde{Q})=D \widetilde{Q} D^{-1}=\widetilde{Q}
\end{aligned}
$$

And furthermore, $\widetilde{Q} \in \overline{\mathrm{co}}\left(\operatorname{LIM}\left(S_{n}\right)_{n \geq 1}\right) \subseteq \overline{\mathrm{co}}\left(\mathrm{E}\left(\mu_{s}\right)\right)$ (by step 2). Note that $\operatorname{LIM}\left(S_{n}\right)_{n \geq 1}$ being compact in a finite dimensional vector space yields $\overline{\operatorname{co}}\left(\operatorname{LIM}\left(S_{n}\right)_{n \geq 1}\right)=\operatorname{co}\left(\operatorname{LIM}\left(S_{n}\right)_{n \geq 1}\right)$. Hence we obtain $\widetilde{Q} \in \mathrm{E}\left(\mu_{s}\right)$ (4.1, step 8), and we have $\widetilde{Q} \in \mathrm{E}\left(\mu_{s}\right) \backslash \mathrm{E}^{0}\left(\mu_{s}\right)$ according to 4.1 step 7. (Cf. the proof of Lemma 14 in [17].) Whence $\left(\sigma_{t}\right) \subseteq \mathrm{D}\left(\mu_{s}\right)-$ if $\left(\sigma_{t}\right)$ is defined by $\sigma_{t}^{o}=e^{t \widetilde{Q}}$, $t \geq 0$.

Replacing $\mathrm{D}\left(\mu_{s}\right)=\mathrm{D}^{l}\left(\mu_{s}\right)$ by $\mathrm{D}^{r}\left(\mu_{s}\right)$ we obtain analogously $\left(\sigma_{t}\right) \subseteq \mathrm{D}^{r}\left(\mu_{s}\right)$, and therefore $\left(\sigma_{t}\right) \subseteq \mathrm{D}^{c}\left(\mu_{s}\right)$ follows. Whence obviously $\left(\sigma_{t}\right)_{t>0} \subseteq \mathrm{DC}\left(\mu_{s}\right)$, $s>0$.

Remarks. a) If $\rho_{\bullet}$ belongs to the decomposability semigroup with commuting cofactors, i.e. $\left(\rho_{t}\right)_{t \geq 0} \subseteq \mathrm{C}(\mu)$ we obtain for all $n \in \mathbb{N}$ and $t \geq 0$ that $\rho_{t} \in \mathrm{C}\left(\mu^{n}\right), \mu^{n}=\nu(n, t) * \rho_{t}\left(\mu^{n}\right)$ with (commuting) cofactors $\nu(n, t) \in$ $\mathcal{M}^{1}(\mathbb{G})$. In fact, we can choose $\nu(n, t)=\nu(1, t)^{n}$ in this case.

b) Note that in 4.2 it is shown that $\sigma_{t}$ belongs to $\mathrm{D}^{l}\left(\mu_{s}\right) \cap \mathrm{D}^{r}\left(\mu_{s}\right)$, but even if we assume $\rho_{\bullet} \subseteq \mathrm{C}(\mu)$ it can not be proved that $\sigma_{t} \in \mathrm{C}\left(\mu_{s}\right)$. This 
is due to the fact that $\widetilde{Q}$ is a limit of convex-combinations of $\left\{i_{D}^{n}(Q)\right\}$, and hence $\sigma_{t}$ belongs to the closed semigroup generated by $\mathrm{C}\left(\mu_{s}\right)$.

In analogy to vector spaces we define for a simply connected nilpotent Lie group

Definition 4.3. Let $\mu \in \mathcal{M}^{1}(\mathbb{G})$. $\mu$ possesses independent marginals if $\mathbb{G}$ is representable as semidirect product $\mathbb{G}=\mathbb{G}_{1} \times \mathbb{G}_{2}$ of closed subgroups where $\mathbb{G}_{1}$ is normal in $\mathbb{G}$, such that $\mu=\mu_{1} \otimes \mu_{2}$ with probabilities $\mu_{i}$ supported by $\mathbb{G}_{i}$.

In particular, let $\pi_{i}$ denote the canonical projections $\mathbb{G} \rightarrow \mathbb{G}_{i}$, then $\pi_{1}, \pi_{2}$ are independent random variables on the probability space $(\mathbb{G}, \mathcal{B}(\mathbb{G}), \mu)$. But in contrast to vector spaces, since $\mathbb{G}$ is not supposed to be Abelian, a priori we do not assume $\mathbb{G}$ to be a direct product, hence $\pi_{1}$ will in general not be a homomorphism. (In fact, here we will always be led to direct splitting $\mathbb{G}=\mathbb{G}_{1} \times \mathbb{G}_{2}$, hence all projections $\pi_{i}$ will turn out to be homomorphisms.)

Proposition 4.4. a) (Cf. [21], Theorem 5) Let $\mu$ be $S$-full and selfdecomposable with contractive $\rho_{\bullet} \subseteq \mathrm{D}(\mu)$. Then $\mu$ is class L, i.e. there exist sequences $\left(\mu_{n}\right)_{n \in \mathbb{N}} \subseteq \mathcal{M}^{1}(\mathbb{G})$ and $\left(b_{n}\right)_{n \in \mathbb{N}}$ such that $\left\{b_{n} \mu_{k}\right\}_{1 \leq k \leq n}$ is infinitesimal and the row products converge to $\mu$. Moreover, $b_{n} b_{n+1}^{-1} \rightarrow$ id. $\left\{b_{n}\right\}$ is called a norming sequence.

b) By construction, $b_{n}=\rho_{\alpha(n)}$ for some sequence $\alpha(n) \rightarrow \infty$, hence if $\rho_{\bullet} \subseteq \mathrm{DC}(\mu)$ then $\left\{b_{n}\right\} \subseteq \mathrm{DC}(\mu)$. A norming sequence $\left\{b_{n}\right\} \subseteq \mathrm{DC}(\mu)$ is called a strong norming sequence.

Proposition 4.5. a) (Cf. [21], Theorem 1, (iii), [10], 3.3.3) Let $\mathbb{G}, \mu$ be as above and let $\left(b_{n}\right)$ be a norming sequence. Assume the existence of a non-trivial idempotent $\varepsilon \in \mathrm{D}(\mu)$. Then $\mathrm{D}_{\varepsilon}(\mu):=\{b \in \mathrm{D}(\mu): \varepsilon b \varepsilon=b\}$ contains a one-parameter semigroup $\left(\rho_{t}\right)_{t \geq 0}$ with $\rho_{0}=\varepsilon$ and an idempotent $\delta \in \mathrm{D}_{\varepsilon}(\mu), \delta \neq \varepsilon$, a limit point of $\left\{\left(\rho_{t}\right): t \rightarrow \infty\right\}$ and thus commuting with $\rho_{t}$ for all $t$.

b) In fact, as the proof (cf. [10], 3.3.3) shows, if $\left\{b_{n}\right\}$ and $\varepsilon$ belong to $\mathrm{D}^{c}(\mu)$ (resp. $\left.\mathrm{DC}(\mu)\right)$ then $\rho_{\bullet}$ and $\delta$ belong to the same semigroup. (See also [21], Remark 1.)

Proposition 4.6. a) If $\operatorname{End}(\mathbb{G})$ contains an idempotent $\varepsilon$ then $\mathbb{G}$ splits semidirectly, $\mathbb{G}=\mathbb{G}_{1} \times \mathbb{G}_{2}$ with $\mathbb{G}_{2}=\varepsilon(\mathbb{G})$ and $\mathbb{G}_{1}=\operatorname{ker} \varepsilon$.

b) If $\varepsilon=\varepsilon^{2} \in \mathrm{D}(\mu)$ then $\mu$ splits, $\mu=\varepsilon(\mu) \otimes \mu(\varepsilon)$ for some $\mu(\varepsilon) \in$ $\mathcal{M}^{1}\left(\mathbb{G}_{2}\right)$, i.e. $\mu$ is decomposed into independent marginals.

c) Moreover, if $\varepsilon \in \mathrm{D}^{c}(\mu)$ then $\mathbb{G}_{1}$ and $\mathbb{G}_{2}$ are direct factors, $\mathbb{G}=\mathbb{G}_{1} \times \mathbb{G}_{2}$. 
[a) Cf. [21], Lemma 4. c) Cf. [21] Lemma 7. ]

b) Cf. the discussion in [21] before Theorem 2 .

In particular, 4.6 applies in the situation of 4.5 .

In the above-mentioned investigation [21] the author obtains (in Theorem 2) for $S$-full class $L$ measures (hence with some norming sequence $\left.\left(b_{n}\right) \subseteq \mathrm{D}(\mu)\right)$ a splitting $\mathbb{G}=\mathbb{G}_{1} \cdots \mathbb{G}_{k}, \mu=\mu_{1} \otimes \cdots \otimes \mu_{k}$ with closed subgroups $\mathbb{G}_{i}$ and selfdecomposable $\mu_{i} \in \mathcal{M}^{1}\left(\mathbb{G}_{i}\right), 1 \leq i \leq k$. The additional assumption $\left(b_{n}\right) \subseteq \mathrm{DC}(\mu)$ leads to a slightly stronger result. First we prove:

Proposition 4.7. Let $\mu$ be $S$-full and class $L$ with strong norming sequence $\left(b_{n}\right) \subseteq \mathrm{DC}(\mu)$. Assume $\varepsilon=\varepsilon^{2} \in \mathrm{DC}(\mu)$. Then $\varepsilon(\mu)$ is selfdecomposable in $\mathcal{M}^{1}(\varepsilon(\mathbb{G}))$, i.e. there exists a contracting semigroup $\sigma \bullet \subseteq \mathrm{DC}(\varepsilon(\mu))$.

Proof. (Cf. [21], proof of Theorem 2). According to $4.6 \mathrm{c}$ ) we obtain a direct splitting $\mathbb{G}=\mathbb{G}_{*} \times \mathbb{G}_{1}^{\prime}, \mu=\mu_{*} \otimes \lambda_{1}$ with $\lambda_{1}=\varepsilon(\mu), \mathbb{G}_{1}^{\prime}=\varepsilon(\mathbb{G})$. It rests to prove selfdecomposability of $\lambda_{1}$.

In view of 4.4 and 4.5 there exists a semigroup $\sigma_{\bullet}^{(1)} \subseteq \mathrm{DC}(\mu)$ with $\sigma_{0}^{(1)}=\varepsilon$ and an idempotent $\varepsilon^{(1)} \neq \varepsilon$ such that $\varepsilon^{(1)}$ is the unit of the compact group $\mathcal{A}^{(1)}:=\operatorname{LIM}\left(\sigma_{t}^{(1)}: t \rightarrow \infty\right)$. Hence in particular we have $\varepsilon^{(1)} \varepsilon=\varepsilon \varepsilon^{(1)}=\varepsilon^{(1)}$ and $\varepsilon^{(1)} \sigma_{\bullet}^{(1)}=\sigma_{\bullet}^{(1)} \varepsilon^{(1)}$. Therefore $\sigma_{\bullet}^{(1)} \subseteq \mathrm{DC}(\varepsilon(\mu))$ follows.

If $\varepsilon^{(1)}$ is trivial, i.e. $\varepsilon^{(1)}(x) \equiv e$ then $\sigma_{\bullet}^{(1)}$ is contracting. (Cf. the proof of Theorem 2 in [21].) If $\varepsilon^{(1)}$ is non-trivial then we proceed as follows:

Let $\mathbb{G}_{1}:=\varepsilon\left(\operatorname{ker}\left(\varepsilon^{(1)}\right)\right), \mathbb{G}_{2}^{\prime}:=\varepsilon^{(1)}(\mathbb{G})$. According to 4.6 we obtain a direct splitting $\mathbb{G}_{1}^{\prime}=\mathbb{G}_{1} \times \mathbb{G}_{2}^{\prime}, \varepsilon(\mu)=\mu_{1} \otimes \lambda_{2}, \lambda_{2}=\varepsilon^{(1)}(\mu)=\varepsilon^{(1)}\left(\lambda_{1}\right)$ with $\mu_{1}$ supported on $\mathbb{G}_{1}$. And as the proof of Theorem 2 in [21] shows, there exist $a_{t} \in \mathbb{G}_{2}^{\prime}$ such that $\left(\left.i_{a_{t}} \sigma_{t}^{(1)}\right|_{\mathbb{G}_{1}}\right) \subseteq \mathrm{D}\left(\varepsilon^{(1)}(\mu)\right)$ is contractive, $i_{a_{t}}$ denoting the inner automorphism $x \mapsto a_{t} x a_{t}^{-1}$. Since the above splitting is direct $\left.i_{a_{t}}\right|_{\mathbb{G}_{1}}$ is trivial, whence $\left.i_{a_{t}} \sigma_{t}^{(1)}\right|_{\mathbb{G}_{1}}=\left.\sigma_{t}^{(1)}\right|_{\mathbb{G}_{1}}=: \rho_{t}^{(1)}$. Thus $\mu_{1}$ is selfdecomposable in $\mathcal{M}^{1}\left(\mathbb{G}_{1}\right)$ with contracting $\rho_{\bullet}^{(1)} \subseteq \mathrm{DC}\left(\mu_{1}\right)$.

Repeating the above construction with $\varepsilon$ replaced by $\varepsilon^{(1)}$, we get $\varepsilon^{(2)}, \mathbb{G}_{3}^{\prime}=$ $\varepsilon^{(2)}(\mathbb{G}), \mathbb{G}_{2}=\varepsilon^{(1)}\left(\operatorname{ker} \varepsilon^{(2)}\right)$ and $\rho_{\bullet}^{(2)}$.

Now if $\varepsilon^{(2)}$ is non-trivial then again repeating the above construction successively we get $\varepsilon^{(i)}, \mathbb{G}_{i+1}^{\prime}=\varepsilon^{(i)}(\mathbb{G}), \mathbb{G}_{i}=\varepsilon^{(i-1)}\left(\operatorname{ker} \varepsilon^{(i)}\right)$ and contracting $\rho_{\bullet}^{(i)}$ in $\mathrm{DC}\left(\mu_{i}\right)$, where $\mathbb{G}_{i}^{\prime}=\mathbb{G}_{i} \times \mathbb{G}_{i+1}^{\prime}, \lambda_{i}=\varepsilon^{(i-1)}(\mu)=\mu_{i} * \lambda_{i+1}$ and $\varepsilon(\mu)=\lambda_{1}=\mu_{1} * \mu_{2} * \cdots * \lambda_{i+1}$. Now observing that $\operatorname{dim}\left(\varepsilon^{(i)}(\mathbb{G})\right)$ is strictly decreasing we obtain finally a trivial idempotent $\varepsilon^{(k)}$, hence a contracting semigroup $\left.\sigma_{\bullet}^{(k)}\right|_{\mathbb{G}_{k}^{\prime}} \subseteq \operatorname{DC}\left(\lambda_{k}\right)$. Then put $\mathbb{G}_{k}^{\prime}=: \mathbb{G}_{k},\left.\sigma_{\bullet}^{(k)}\right|_{\mathbb{G}_{k}}=: \rho_{\bullet}^{(k)}$ and $\lambda_{k}=: \mu_{k}$. 
Since the splitting is direct,

$$
\varepsilon(\mathbb{G})=\mathbb{G}_{1} \times \cdots \times \mathbb{G}_{k} \ni\left(x_{1}, \ldots x_{k}\right) \mapsto\left(\sigma_{\bullet}^{(1)} x_{1}, \ldots \sigma_{\bullet}^{(k)} x_{k}\right)
$$

defines a semigroup of automorphisms $\rho_{\bullet} \subseteq \operatorname{Aut}(\varepsilon(\mathbb{G}))$ which is obviously contracting and we have $\rho_{\bullet} \subseteq \mathrm{DC}(\varepsilon(\mu))$.

Corollary 4.8. Let $\mu$ be $S$-full and let $\left(b_{n}\right)$ be a strong norming sequence in $\mathrm{DC}(\mu)$. Then $\mu$ is selfdecomposable and there exists a contracting semigroup $\rho_{\bullet} \subseteq \mathrm{DC}(\mu)$.

[ The proof follows directly from Proposition 4.7, if we replace $\varepsilon$ by the identity automorphism, hence $\varepsilon(\mu)=\mu$ is selfdecomposable. ]

We are ready to prove the main result of this section: Combining the ideas of the proof of 4.7 ([21], Theorem 2) and of [17], Theorem 15, we obtain:

Theorem 4.9. Let $\mu \in \mathcal{M}^{1}(\mathbb{G})$ be full and $(a, \alpha)$-semistable, let again $\mu_{\bullet}$ denote the semistable convolution semigroup with $\mu_{1}=\mu$. Assume furthermore that $\left(\rho_{t}\right)_{t \in \mathbb{R}}$ is contracting and $\left(\rho_{t}\right)_{t \geq 0} \subseteq \mathrm{DC}(\mu)$, i.e.

$$
\mu^{n}=\nu(t, n) * \rho_{t}\left(\mu^{n}\right)=\rho_{t}\left(\mu^{n}\right) * \nu^{\prime}(t, n), t \geq 0, n \in \mathbb{N} .
$$

Then there exists a contractive one-parameter semigroup $\left(\sigma_{t}\right)_{t \in \mathbb{R}_{+}} \subseteq \mathrm{DC}\left(\mu_{s}\right)$ for all $s \geq 0$, such that $\left(*^{\prime}\right) \quad a \sigma_{t}=\sigma_{t}$ a for all $t \geq 0$.

Proof. 1. In view of 4.2 there exists $Q_{1} \in \mathrm{E}\left(\mu_{s}\right) \backslash \mathrm{E}^{0}\left(\mu_{s}\right)$, for $s \geq 0$, commuting with $a^{o}$, i.e. $Q_{1}$ generates a one-parameter semigroup $\left(\sigma_{t}\right) \subseteq$ $\mathrm{DC}\left(\mu_{s}\right), s \geq 0$, commuting with $a^{o}$. If $\left(\sigma_{t}\right)_{t \geq 0}$ is contractive the proof is done.

Else we repeat more or less the proof of 4.7:

2. If $\left(\sigma_{t}\right)_{t \geq 0}$ is not contractive then the set of accumulation points $\mathcal{C}:=$ $\operatorname{LIM}\left\{\sigma_{t}: t \rightarrow \infty\right\}$ is a compact group, let $\varepsilon$ denote the unit element of $\mathcal{C}$. Since $Q_{1} \notin \mathrm{E}^{0}(\mu)$ we have $\varepsilon \neq \mathrm{id}$. Hence Proposition 4.6 applies and yields a direct splitting $\mathbb{G}=\mathbb{G}_{1} \times \mathbb{G}_{2}$, furthermore $\mu=\lambda_{1} \otimes \lambda_{2}$ with $\lambda_{i} \in \mathcal{M}^{1}\left(\mathbb{G}_{i}\right)$ and by construction $\left.\sigma_{t}\right|_{\mathbb{G}_{1}}$ is contractive. $\mathbb{G}_{i}$ are $a$-invariant since $\sigma_{\bullet}$ is commuting with $a$. Let $\pi_{i}$ denote the projections to $\mathbb{G}_{i}$, then $\varepsilon=\pi_{2}$, $\mathbb{G}_{1}=\operatorname{ker} \varepsilon$ and $\lambda_{i}=\pi_{i}(\mu)$. Also, $a \varepsilon=\varepsilon a$ and hence $a\left(\mathbb{G}_{i}\right)=\mathbb{G}_{i}$ for each $i$.

The second part of the proof of 4.7 (resp. [21], Theorem 2) yields that the first factor $\lambda_{1}$ is selfdecomposable on $\mathbb{G}_{1}$ : There exists a contractive semigroup $\left(\sigma_{\bullet}^{(1)}\right)_{t \geq 0} \subseteq \operatorname{DC}\left(\lambda_{1}\right)$, where $\sigma_{\bullet}^{(1)}:=\left.\sigma_{\bullet}\right|_{\mathbb{G}_{1}}$, commuting with $\left.a\right|_{\mathbb{G}_{1}}$.

3. In particular, if $\mu$ is supposed to have no independent marginals we are led to step 1 , the proof is done. 
4. Concerning $\lambda_{2}$ we conclude by 4.7 that $\lambda_{2}=\varepsilon(\mu)$ is selfdecomposable, i.e. there exists a contracting semigroup $\rho_{\bullet}^{(1)} \subseteq \mathrm{DC}\left(\lambda_{2}\right)$. Also, since $a \varepsilon=\varepsilon a$, $\lambda_{2}$ is $\left(\left.a\right|_{\mathbb{G}_{2}}, \alpha\right)$-semistable.

5. Now applying the previous steps 1 to 4 to $\mathbb{G}_{2}, \lambda_{2},\left.a\right|_{\mathbb{G}_{2}}$ and $\rho_{\bullet}^{(1)}$ and repeating this construction we obtain finally decompositions $\mathbb{G}=\mathbb{G}_{1} \times \cdots \times$ $\mathbb{G}_{n}, \mu=\lambda_{1} \otimes \cdots \otimes \lambda_{n}, \lambda_{i} \in \mathcal{M}^{1}\left(\mathbb{G}_{i}\right)$ selfdecomposable with contractive $\left.\sigma_{\bullet}^{(i)}\right|_{\mathbb{G}_{i}} \subseteq \operatorname{DC}\left(\lambda_{i}\right) \subseteq \operatorname{Aut}\left(\mathbb{G}_{i}\right)$ which commute with $\left.a\right|_{\mathbb{G}_{i}}$ for all $i$. As above, since the factors $\mathbb{G}_{i}$ are direct, $x=\left(x_{1}, \ldots x_{n}\right) \mapsto\left(\sigma_{\bullet}^{(1)}\left(x_{1}\right), \ldots, \sigma_{\bullet}^{(n)}\left(x_{n}\right)\right)$ defines a one-parameter semigroup $\left(\sigma_{\bullet}\right) \subseteq \operatorname{Aut}(\mathbb{G})$, which is contracting and commuting with $a$ by construction and belongs to $\mathrm{DC}(\mu)$.

Since for each $t, a \sigma_{t}=\sigma_{t} a$, as in step 1 of the proof of Proposition 4.2 we obtain $\sigma_{t} \in \mathrm{DC}\left(\mu_{\alpha^{n}[k(n) s]}\right)$ for each $n \in \mathbb{N}$ and $s \geq 0$, where $k(n)=\left[\alpha^{-n}\right]$. Hence $\sigma_{t} \in \mathrm{DC}\left(\mu_{s}\right)$ for all $s, t \geq 0$ follows.

In view of [21], Theorem 4, we obtain for class $L$-laws

Corollary 4.10. Let $\mu \in \mathcal{M}^{1}(\mathbb{G})$ be full and $(a, \alpha)$-semistable. Furthermore, assume that there exist $\left(\mu_{n}\right)_{n \in \mathbb{N}} \subseteq \mathcal{M}^{1}(\mathbb{G})$ and $\left(b_{n}\right)_{n \in \mathbb{N}} \subseteq \operatorname{Aut}(\mathbb{G})$ such that

(†) $\quad \mu=\lim _{n \geq 1} b_{n}\left(\mu_{1} * \cdots * \mu_{n}\right)$

(††) $\quad \mu_{n} * \mu_{k}=\mu_{k} * \mu_{n}$, all $n, k \in \mathbb{N}$ and

(††) the array $\left\{b_{n}\left(\mu_{k}\right): 1 \leq k \leq n\right\}$ is infinitesimal.

Then there exists a contracting group $\left(\sigma_{t}\right)_{t \in \mathbb{R}} \subseteq$ Aut $(\mathbb{G})$ such that $\left(\sigma_{t}\right)_{t \geq 0} \subseteq \mathrm{DC}\left(\mu_{s}\right), s \geq 0$, and $\left(*^{\prime}\right) \quad a \sigma_{t}=\sigma_{t} a, t \geq 0$.

In $[21]$, Theorem 4 it is shown that under the assumptions $(\dagger),(\dagger \dagger),(\dagger \dagger \dagger)$ there exists a contracting group $\left(\rho_{t}\right)_{t \in \mathbb{R}}$ such that $\left(\rho_{t}\right)_{t \geq 0} \subseteq \mathrm{DC}(\mu)$, whence by 4.4 a strong norming sequence exists and hence 4.8 and 4.9 apply. $]$

And by [21], Theorem 5 we obtain a partial converse result:

Corollary 4.11. Let $\mu$ be $S$-full, $(a, \alpha)$-semistable and selfdecomposable with contractive semigroup $\left(\rho_{\bullet}\right) \subseteq \mathrm{C}(\mu)$. Then there exists a norming sequence $\left(b_{n}\right) \subseteq \operatorname{Aut}(\mathbb{G})$, a sequence $\left(\mu_{k}\right) \subseteq \mathcal{M}^{1}(\mathbb{G})$ such that $(\dagger),(\dagger \dagger)$, (††) hold.

【 The semistable law $\mu$ is embeddable into a convolution semigroup $\mu_{\bullet}$ (uniquely defined, cf. [4]). According to [15] the convolution operators corresponding to embeddable laws $\mu_{t}$ are injective, $t \geq 0$. Whence [21], Theorem 5 yields the existence of a commutative triangular system $\left\{b_{n} \mu_{k}\right\}$. ]

Acknowledgement. The authors would like to thank the referee for pointing out an error in Section 4 of the first version. 


\section{References}

[1] Dani, S. G., Shah, R., Contractible measures and Lévy's measures on Lie groups, in: "Probabilities on Algebraic Structures" (G. Budzban, Ph. Feinsilver and A. Mukherjea, Eds.), Contemp. Math. 261 (2000), 3-14.

[2] Hazod, W., Remarks on convergence of types theorems for finite dimensional vector spaces, Publ. Math. Debrecen 50 (1997), 197-219.

[3] Hazod, W., Stetige Faltungshalbgruppen von Wahrscheinlichkeitsmaßen und erzeugende Distributionen, Lecture Notes in Math. 595 (1977), Springer, BerlinHeidelberg-New York.

[4] Hazod, W., On some convolution semi- and hemigroups appearing as limit distributions of normalized products of group-valued random variables, Conference on infinite dimensional harmonic analysis (Marseille, 1997), World Sci. Publishing, River Edge, 1998, 104-121.

[5] Hazod, W., Nobel, S., Convergence of types theorem for simply connected nilpotent Lie groups, "Probability measures on groups, IX" (Oberwolfach, 1988), Lecture Notes in Math. 1375 (1989), Berlin-Heidelberg-New York, Springer. 99-106.

[6] Hazod, W., Scheffler, H-P., The domains of partial attraction of probabilities on groups and on vectorspaces, J. Theoret. Probab. 6 (1993), 175-186.

[7] Hazod, W., Siebert, E., Stable probability measures on Euclidean spaces and on locally compact groups: Structural properties and limit theorems, In preparation (Kluwer).

[8] Heyer, H., Probability Measures on Locally Compact Groups, Springer, Berlin-Heidelberg-New York, 1977.

[9] Hochschild, G., The Structure of Lie Groups, Holden Day, Inc., San Francisco, London, Amsterdam, 1965.

[10] Jurek, Z., Mason, J. D., Operator Limit Distributions in Probability Theory, Wiley Series in Probability and Mathematical Statistics: Probability and Mathematical Sciences, John Wiley \& Son, Inc., New York, 1993.

[11] Krakowiak, W., Zero-one laws for A-decomposable measures on Banach spaces, Colloq. Math. 43 (1980), 351-363.

[12] Krengel, U., Ergodic Theorems, de Gruyter Studies in Mathematics 6 (1985), de Gruyter \& Co., Berlin-New York.

[13] Kunita, H. Infinitesimal generators of nonhomogeneous convolution semigroups on Lie groups, Osaka J. Math. 34 (1997), 233-264.

[14] Kunita, H., Stochastic processes with independent increments on a Lie group and their self-similar properties, "Stochastic Differential and Difference Equations" (Györ, 1996), Progr. Systems Control Theory 23 (1997), 183-201.

[15] Kunita, H., Analyticity and injectivity of convolution semigroups on Lie groups, J. Funct. Anal. 165 (1999), 80-100.

[16] Loève, M., Nouvelles classes de lois limites, Bull. Soc. Math. France 73 (1945), $107-126$.

[17] Łuczak, A. Operator-semistable operator Lévy's measures on finite dimensional vector spaces, Probab. Theory Related Fields 90 (1991), 317-340.

[18] Nobel, S., Limit theorems for probability measures on simply connected nilpotent Lie groups, J. Theoret. Probab. 4 (1991), 261-284.

[19] Parthasarathy, K. R., Probability Measures on Metric Spaces, Academic Press, New York, 1967.

[20] Shah, R., Semistable measures and limit theorems on real and p-adic groups, Monatsh. Math. 115 (1993), 191-213. 
[21] Shah, R., Selfdecomposable measures on simply connected nilpotent Lie groups, J. Theoret. Probab. 13 (2000), 65-83.

[22] Siebert, E., Supports of holomorphic convolution semigroups on a locally compact group, Arch. Math. (Basel) 36 (1981), 423-433.

[23] Siebert, E., Strongly operator-decomposable probability measures on separable Banach spaces, Math. Nachr. 154 (1991), 315-326.

[24] Siebert, E., Operator-decomposability of Gaussian measures on separable Banach spaces, J. Theoret. Probab. 5 (1992), 333-347.

[25] Urbanik, K., Lévy's probability measures on Euclidean spaces, Studia Math. 44 (1972), 119-148.

W. HAZOD

DEPARTMENT OF MATHEMATiCS

UNIVERSITY OF DORTMUND

D-44221 DorTMund

Germany

HAZOD@MATH.UNI-DORTMUND.DE
RIDDHI SHAH

School of Mathematics

TAta Institute

of Fundamental Research

HOMI BHABHA ROAD

BOMBAY 400 005, INDIA

RIDDHI@MATH.TIFR.RES.IN 